\title{
Three-Dimensional Numerical Study of the Effect of Protective Barrier on the Dispersion of the Contaminant in a Building
}

\author{
Chemseddine Maatki ${ }^{1,2}$
}

1 Department of Mechanical and Industrial Engineering, College of Engineering, Imam Mohammad Ibn Saud Islamic University, Riyadh 11432, Saudi Arabia; casmaatki@imamu.edu.sa

2 Laboratory of Metrology and Energy System, University of Monastir, Monastir City 5000, Tunisia

Citation: Maatki, C.

Three-Dimensional Numerical Study of the Effect of Protective Barrier on the Dispersion of the Contaminant in a Building. Mathematics 2021, 9, 1125. https://doi.org/10.3390/ math9101125

Academic Editors: Theodore E. Simos and Charampos Tsitouras

Received: 3 April 2021

Accepted: 13 May 2021

Published: 16 May 2021

Publisher's Note: MDPI stays neutral with regard to jurisdictional claims in published maps and institutional affiliations.

\begin{abstract}
The finite volume method and potential-vorticity vector formalism in their three-dimensional form were used to numerically study the impact of an adiabatic and impermeable vertical barrier on the dispersion of a local aero-contaminant due to the double-diffusive Rayleigh-Benard convection inside a cubic container. Different governing parameters such as the Rayleigh number, buoyancy ratio and barrier height were analyzed for $L e=1.2$ and $P r=0.7$, representing an air-contaminant mixture. The potential-vector-vorticity formalism in the three-dimensional form allowed the elimination of the pressure terms appearing in the Navier-Stokes equations. It was found that the heat and mass transfer as well as the effectiveness of the barrier in reducing contaminant dispersion are strongly influenced by the buoyancy ratio, the barrier size and the Rayleigh number. In addition, the barrier effectiveness is more than $70 \%$ for a height of half the building height.
\end{abstract}

Keywords: numerical study; finite volume method; 3D vorticity-potential vector; double diffusiveconvection; Rayleigh-Benard; protective barrier; aero-contaminant dispersion

\section{Introduction}

Improving air quality has been one of the most important objectives of scientific research in recent years, particularly in view of the spread of viruses. The assessment of air quality is based on the measurement and identification of all types of air pollutants, without attempting to distinguish between pollutants of natural origin and those resulting from human activity or virus circulation. An air monitoring system or solution can be used to manage exposure to air pollutants when the source strength is local and moderate, such as smoke, dust or sprays. Several researchers have investigated air quality monitoring using analytical, experimental and numerical models.

Many dangerous chemical pollutants are present in the indoor environment that adversely affect people's comfort and health [1]. Formaldehyde and volatile organic compounds (VOCs) are the main pollutants emitted from building materials that humans may be exposed to through inhalation [2,3]. Several authors have investigated the emission characteristics of formaldehyde and volatile organic compounds from building materials and the associated health risks in order to achieve an effective source control and create a sustainable built environment. The researchers focused on creating analytical or numerical solutions to predict the concentration of pollutants in a room or indoor environment based on the mass transfer analysis [4-8]. Wiglusz et al. [9] examined an experimental study of the effect of temperatures on emissions of formaldehyde and volatile organic compounds (VOCs) from relevant plates. They showed that at 23 and $29^{\circ} \mathrm{C}$, there are no formaldehyde emissions and they observed very low emissions of VOCs. But at $50{ }^{\circ} \mathrm{C}$, the authors noticed high initial emissions of formaldehyde and VOCs, which decreased over time. Xiong et al. [10] theoretically studied the overall effect of temperature and relative humidity on the emission rate of pollutants from building materials. In addition, the authors proposed a new approach to assess the impact on human health due to pollutants emitted at varying temperatures and relative humidity and concluded that there is 
a correlation between the likelihood of human cancer (HCP) and environmental factors. Deng et al. [11] numerically examined the double diffusive mixed convection in a bidimensional cavity with contaminant. They noticed that the flow structure due to a doublediffusive convection can detect indoor airflow, heat transfer structures and pollutants. Yu-Shu et al. [12] numerically studied the forced and mixed convection in a cavity filled with a porous medium with a local contaminant source. They found a critical factor in terms of the Reynolds number and Darcy number for a flow structure transition from vortex-free to multi-vortexes. Zhao et al. [13] numerically studied the double diffusive convection in the cavity saturated by porous media with local heat and a contaminant source. The authors concluded that the permeability of porous medium strongly affects the heat and mass transfer in the cavity. A numerical investigation of double diffusive convection in the enclosure with local $\mathrm{CO}_{2}$ source was studied by Arellano et al. [14]. The authors noted that the Nusselt number is directly affected by the location of the contaminant's source. Li et al. [15] numerically studied the double diffusive convection and radiation effect on the dispersion of gas contaminant in a cavity. They noticed that surface radiation represents about $37-71 \%$ of the heat transfer from the heat sources. They also remarked that the diffusion of the contaminants is dependent on the temperature. Consequently, the contaminants exhibit different distribution patterns in the air.

Several numerical and mathematical methods have been used in recent years to solve the convection-diffusion differential equation. Abolhasani et al. [16] introduced a resolution method based on the variational iteration method (VIM). Geiser et al. [17] applied parallel iterative splitting methods, allowing an acceleration of the solver and subsequently a reduction of the computation time.

Emissions and dispersion of viruses or other volatile organic compounds (VOCs) inside buildings is a major problem that has needed to be addressed in recent years. This is because airflow near walls and under the influence of thermal gradient plays an important role in the transport of pollutants from the emitting source to the ambient air and because recirculating air in forced convection can be dangerous in terms of increasing the dispersion of pollutants. Therefore, for these reasons, an effective prediction of the manner in which contaminants are emitted and dispersed in natural convection allows us to control their hazards.

The main objective of this work is to analyze the flow pattern of the natural 3D doublediffusive convection in an air-filled building with a local source of contaminants, using the finite volume method and the vector potential-vorticity formalism in three-dimensional form, to analyze the mode of dispersion of the contaminants, and to examine the efficiency of the placement of a barrier to avoid contamination of the whole building volume for different Rayleigh numbers and buoyancy ratios.

\section{Physical Configuration and Governing Equations}

In this section, the physical configuration, the assumptions and the governing equations are described. The studied configuration, illustrated in Figure 1, represents an air-filled cavity with a local source of contamination located at the bottom surface. The air-contaminant mixture in the cavity is characterized by the Lewis and Prandtl numbers $L e=1.2$ and $\operatorname{Pr}=0.72$, respectively. The bottom wall is hot $\left(\mathrm{T}_{\mathrm{H}}^{\prime}\right)$ and the top wall is cold $\left(\mathrm{T}_{\mathrm{c}}^{\prime}{ }_{\mathrm{c}}\right)$. However, the source of contamination is located in half of the surface of the bottom wall with a high concentration $\left(C_{h}^{\prime}\right)$. The other half of the bottom wall and the top wall have a zero concentration $\left(C_{1}^{\prime}\right)$. The remaining walls (vertical lateral sides) are considered adiabatic, impermeable. The cavity is a cubic of width L. The impact of the insertion of an adiabatic, impermeable vertical barrier, positioned in the middle of the bottom wall, on the dispersion of the contaminant in the whole cavity was investigated. The effect of the height $\left(\mathrm{H}^{\prime}\right)$ of this wall as well as the ratio of the volume forces $(\mathrm{N})$ and the Rayleigh number $(R a)$ were studied, while the thickness of the barrier was fixed at $0.05 \times \mathrm{L}$. 


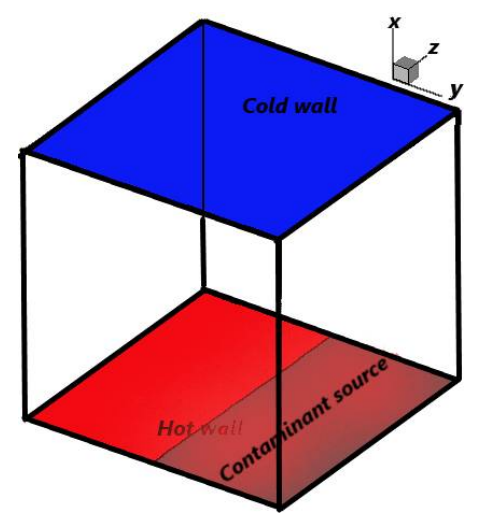

(a)

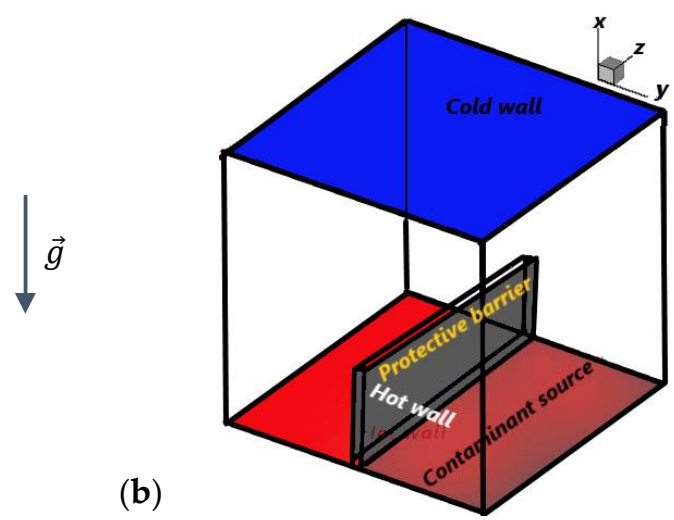

Figure 1. Physical model of the building: (a) Reference model without barrier; (b) Building with barrier.

In this study, the following assumptions were adopted to simplify the formulation of the mathematical model:

- $\quad$ The fluid in the cavity is assumed to be Newtonian and incompressible.

- The flow is assumed to be laminar.

- Heat transfer by radiation is negligible.

- There is no heat or mass source and no chemical reaction

- $\quad$ Soret and Dufour effects are negligible

- The thermo-physical properties of the fluid are assumed to be constant and the Boussinesq approximation is adopted.

The last approximation assumes that the density is constant in all terms of the transfer equations except in the gravitational term, where it varies linearly with temperature and concentration by the following relation:

$$
\rho=\rho_{0}\left[1-\beta_{T}\left(T^{\prime}-T_{0}\right)-\beta_{C}\left(C^{\prime}-C_{0}\right)\right]
$$

where $T^{\prime}$ is the temperature of the fluid mixture and $C^{\prime}$ is the concentration of the contaminant at a given point in the cavity. $\rho_{0}, T_{0}$ and $C_{0}$ are respectively the reference density, temperature and concentration. $\beta_{T}$ and $\beta_{C}$ represent the thermal and solutal expansion coefficients of the fluid. They are defined by:

$$
\beta_{T}=-\frac{1}{\rho_{0}}\left(\frac{\partial \rho}{\partial T^{\prime}}\right)_{P^{\prime}, C^{\prime}} \text { and } \beta_{C}=-\frac{1}{\rho_{0}}\left(\frac{\partial \rho}{\partial C^{\prime}}\right)_{P^{\prime}, T^{\prime}} \text {. }
$$

The fluid motion in the cavity is induced by density variations due to temperature and concentration gradients. Considering the simplifying assumptions, the general equations governing the fluid flow in double diffusion convection are, respectively, the continuity equation, the conservation of momentum, the conservation of energy and the conservation of mass, which can be written in the following vector form:

Continuity

$$
\nabla \vec{V}^{\prime}=0
$$

Momentum

$$
\begin{aligned}
& \frac{\partial \vec{V}^{\prime}}{\partial t^{\prime}}+\left(\vec{V} \times{ }^{\prime} \vec{\nabla}\right) \vec{V}^{\prime}= \\
& \begin{aligned}
\text { Energy } & -\frac{1}{\rho_{0}} \vec{\nabla} P^{\prime}+v \Delta \vec{V}^{\prime}+\left[1-\beta_{T}\left(T^{\prime}-T_{0}\right)-\beta_{C}\left(C^{\prime}-C_{0}\right)\right] \vec{g} \\
\text { Mass } & \frac{\partial T^{\prime}}{\partial t^{\prime}}+\vec{V}^{\prime} \times \nabla T^{\prime}=\alpha \Delta T^{\prime} \\
& \frac{\partial C^{\prime}}{\partial t^{\prime}}+\vec{V}^{\prime} \times \nabla C^{\prime}=D \Delta C^{\prime} .
\end{aligned}
\end{aligned}
$$




\section{Resolution Method Based on the Vorticity-Potential Vector Formalism and Non-Dimensioning of Equations}

To solve the Navier-Stokes equations, vector potential-vorticity formalism is used, which in a three-dimensional configuration eliminates the pressure term from the momentum equation. This method of resolution was adopted by Mallinson and de Vahl Davis [18] and Wakashima and Saitoh [19]. The vector potential and vorticity are defined by the following two relations, respectively:

$$
\vec{\omega}^{\prime}=\vec{\nabla} \times \vec{V}^{\prime} \text { and } \vec{V}^{\prime}=\vec{\nabla} \times \vec{\psi}^{\prime}
$$

The dimensionless formulation of the problem is performed by defining a dimensionless space variable, dimensionless time, dimensionless velocity, vector potential, vorticity, dimensionless temperature and concentration as:

$$
\begin{array}{cccc}
X=x / L & Y=y / L & Z=z / L & H=H^{\prime} / L \\
\omega=\alpha \omega^{\prime} / L^{2} \quad t=\alpha t^{\prime} / L^{2} & V=L V^{\prime} / \alpha \quad \psi=\psi^{\prime} / \alpha \\
T=\frac{T^{\prime}-T_{c}^{\prime} c}{T^{\prime} H^{\prime}-T_{c}} & C=\frac{C^{\prime}-C^{\prime} l}{C^{\prime}{ }_{h}-C_{l}^{\prime}} .
\end{array}
$$

After using the dimensionless variables, the system of equations governing the problem becomes:

$$
\begin{gathered}
\frac{\partial V_{x}}{\partial X}+\frac{\partial V_{y}}{\partial Y}+\frac{\partial V_{z}}{\partial Z}=0 \\
-\vec{\omega}=\nabla^{2} \vec{\psi}
\end{gathered}
$$

$$
\begin{gathered}
\frac{\partial \vec{\omega}}{\partial t}+(\vec{V} \times \vec{\nabla}) \vec{\omega}-(\vec{\omega} \times \vec{\nabla}) \vec{V}=\operatorname{Pr} \times \nabla^{2} \vec{\omega}+\operatorname{RaPr}\left[\frac{\partial T}{\partial z}, 0,-\frac{\partial T}{\partial x}\right]-\operatorname{RaPrN}\left[\frac{\partial C}{\partial z}, 0,-\frac{\partial C}{\partial x}\right] \\
\frac{\partial T}{\partial t}+\vec{V} \times \nabla T=\nabla^{2} T \\
\frac{\partial C}{\partial t}+\vec{V} \times \vec{\nabla} C=\frac{1}{L e} \vec{\nabla}^{2} C .
\end{gathered}
$$

The dimensionless parameters appearing in the equations are:

$$
N=\frac{\beta_{C}\left(C^{\prime}{ }_{h}-C^{\prime}{ }_{l}\right)}{\beta_{T}\left(T^{\prime}{ }_{H}-T^{\prime}{ }_{c}\right)} \quad R a=\frac{g \beta_{T}\left(T^{\prime}{ }_{h}-T^{\prime}{ }_{c}\right) L^{3}}{v \alpha} \quad \operatorname{Pr}=\frac{v}{\alpha} \quad L e=\frac{\alpha}{D} .
$$

$N$ represents the ratio of the solutal volume forces to the thermal volume forces, also called the buoyancy ratio. $R a, P r$ and $L e$ represent the thermal Rayleigh number, Prandtl number and Lewis number, respectively.

The local Nusselt and Sherwood numbers are written as follows:

$$
N u=\left.\frac{\partial T}{\partial x}\right|_{x=0,1} \quad S h=\left.\frac{\partial C}{\partial x}\right|_{x=0,1} .
$$

The average Nusselt and Sherwood numbers on the walls are:

$$
\bar{N} u=\int_{0}^{1} \int_{0}^{1} N u \times \partial y \partial z \quad \bar{S} h=\int_{0}^{1} \int_{0}^{1} S h \times \partial y \partial z .
$$

The boundary conditions are as follows:

Temperature : $\quad T=1$ at $X=0, T=0$ at $x=1, \frac{\partial T}{\partial n}=0$ on the remaining walls (adiabatic) 
Concentration: $\quad C=1$ at $X=0, Y>0.5, C=0$ at $X=1$ and $X=0, Y<0.5, \frac{\partial C}{\partial n}=0$ on the other walls (impermeable)

$$
\begin{gathered}
\omega_{x}=0, \omega_{y}=-\frac{\partial V_{z}}{\partial X}, \omega_{z}=\frac{\partial V_{y}}{\partial X} \text { at } X=0 \text { and } 1 \\
\omega_{x}=\frac{\partial V_{z}}{\partial Y}, \omega_{y}=0, \omega_{z}=-\frac{\partial V_{x}}{\partial Y} \text { at } Y=0 \text { and } 1 \\
\omega_{x}=-\frac{\partial V_{y}}{\partial Z}, \omega_{y}=\frac{\partial V_{x}}{\partial Z}, \omega_{z}=0 \text { at } Z=0 \text { and } 1 \\
\frac{\partial \psi_{x}}{\partial X}=\psi_{y}=\psi_{z}=0 \text { at } X=0 \text { and } 1 \\
\psi_{x}=\frac{\partial \psi_{y}}{\partial Y}=\psi_{z}=0 \text { at } Y=0 \text { and } 1 \\
\psi_{x}=\psi_{y}=\frac{\partial \psi_{z}}{\partial Z}=0 \text { at } Z=0 \text { and } 1
\end{gathered}
$$

Velocity: $\quad V_{x}=V_{y}=V_{z}=0$ On all walls.

The boundary conditions of the adiabatic and impermeable barrier are:

$$
V_{x}=V_{y}=V_{z}=0, \frac{\partial T}{\partial n}=0, \frac{\partial C}{\partial n}=0 .
$$

\section{Numerical Modeling, Discretization, Grid Sensitivity Study and Code Verification}

\subsection{Finite Volume Method}

The numerical code developed during this work was based on the finite volume method. This method consists of subdividing the three-dimensional computational domain into a number of control volumes [20]. The dependent variable considered is calculated at each of these points. The algebraic equations defined at these nodes are obtained by integrating the conservation equations through the control volumes for each node.

\subsection{Meshing}

As shown in Figure 2, a rectangular mesh was considered with $N x$ nodes on the $(x)$ axis, $N y$ nodes on the $(y)$ axis and $N z$ nodes on the $(z)$ axis.

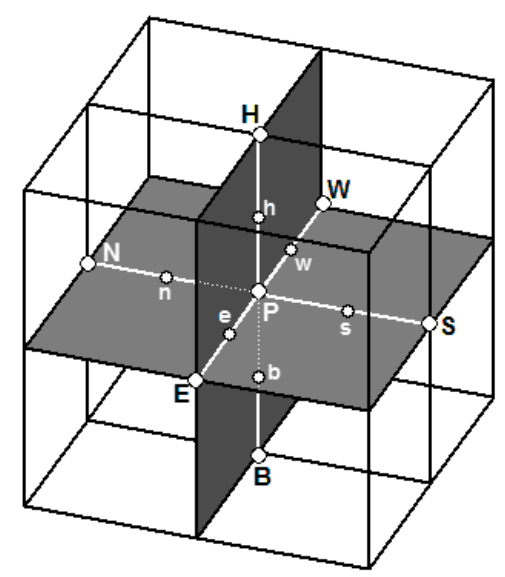

Figure 2. Schematic view of the mesh.

The discrete variables $\mathrm{t}, x$ and $y$ are written as follows:

- $\quad i$ : node index on the $(x)$ axis

- $\quad j$ : node index on the $(y)$ axis

- $\quad k$ : node index on the $(z)$ axis 


$$
\begin{aligned}
& x_{1}=0, x_{2}=0+\frac{\Delta x}{2}, i=3, N x-1, x_{i}=0+(i-1) \Delta x \text { and } x_{N x}=1 \\
& y_{1}=0, y_{2}=0+\frac{\Delta y}{2}, j=3, N y-1, y_{j}=0+(j-1) \Delta y \text { and } y_{N y}=1 \\
& z_{1}=0, z_{2}=0+\frac{\Delta z}{2}, k=3, N z-1, z_{k}=0+(k-1) \Delta z \text { and } z_{N z}=1
\end{aligned}
$$

- $\Delta x$ is the space step size on the $(x)$ axis

- $\Delta y$ is the space step size on the $(y)$ axis

- $\Delta z$ is the space step size on the $(z)$ axis

- And $\Delta t$ is the time step

$\Delta x, \Delta y$ and $\Delta z$ were defined by the following relations:

$$
\Delta x=\frac{1}{N x-1}, \Delta y=\frac{1}{N y-1} \text { and } \Delta z=\frac{1}{N z-1} .
$$

\subsection{Equations Discretization}

The transfer Equations (11)-(13) can be written in the following general form:

$$
\frac{\partial \Phi}{\partial t}+\frac{\partial}{\partial x}\left(V_{x} \cdot \Phi-\Gamma_{\Phi} \frac{\partial \Phi}{\partial x}\right)+\frac{\partial}{\partial y}\left(V_{y} \cdot \Phi-\Gamma_{\Phi} \frac{\partial \Phi}{\partial y}\right)+\frac{\partial}{\partial z}\left(V_{z} \cdot \Phi-\Gamma_{\Phi} \frac{\partial \Phi}{\partial z}\right)=S_{\Phi}
$$

with $\Phi: \mathrm{T}, \mathrm{C}, \omega_{x}, \omega_{y}$ or $\omega_{z}, \Gamma_{\Phi}$ : dimensionless coefficient and $S_{\Phi}$ : source term.

Table 1 summarizes all the transfer equations, momentum, energy and mass.

Table 1. Overview of the dimensionless equations.

\begin{tabular}{ccc}
\hline $\boldsymbol{\Phi}$ & $\boldsymbol{\Gamma}_{\boldsymbol{\Phi}}$ & $\mathbf{S}_{\boldsymbol{\Phi}}$ \\
\hline$\omega_{x}$ & $P r$ & $\omega_{x} \frac{\partial V_{x}}{\partial X}+\omega_{y} \frac{\partial V_{x}}{\partial Y}+\omega_{z} \frac{\partial V_{x}}{\partial Z}+\operatorname{RaPr} \frac{\partial T}{\partial Z}$ \\
$\omega_{y}$ & $P r$ & $\omega_{x} \frac{\partial V_{y}}{\partial X}+\omega_{y} \frac{\partial V_{y}}{\partial Y}+\omega_{z} \frac{\partial V_{y}}{\partial Z}$ \\
$\omega_{z}$ & $P r$ & $\omega_{x} \frac{\partial V_{z}}{\partial X}+\omega_{y} \frac{\partial V_{z}}{\partial Y}+\omega_{z} \frac{\partial V_{z}}{\partial Z}-\operatorname{RaPr} \frac{\partial T}{\partial Z}$ \\
$\mathrm{~T}$ & 1 & 0 \\
$\mathrm{C}$ & $\frac{1}{L e}$ & 0 \\
\hline
\end{tabular}

Equation (27) can be written as:

$$
\frac{\partial \Phi}{\partial t}+\frac{\partial L_{x}}{\partial X}+\frac{\partial L_{y}}{\partial Y}+\frac{\partial L_{z}}{\partial Z}=S_{\Phi}
$$

where $L_{x}, L_{y}$ and $L_{z}$ are the total convection and diffusion fluxes in the $x, y$ and $z$ directions, respectively, and are defined by:

$$
L_{x}=V_{x} \Phi-\Gamma_{\Phi} \frac{\partial \Phi}{\partial X}, L_{y}=V_{y} \Phi-\Gamma_{\Phi} \frac{\partial \Phi}{\partial Y} \text { and } L_{z}=V_{z} \Phi-\Gamma_{\Phi} \frac{\partial \Phi}{\partial Z} .
$$

The integration of Equation (27) on a control volume gives (Patankar [21]):

$$
\left(\Phi_{p}-\Phi_{p}^{\circ}\right) \Delta x \Delta y \Delta z+\left(L_{x e}-L_{x w}\right) \Delta y \Delta z \Delta t+\left(L_{y n}-L_{y s}\right) \Delta x \Delta z \Delta t+\left(L_{z h}-L_{z b}\right) \Delta x \Delta y \Delta t=\bar{S} \Delta x \Delta y \Delta z \Delta t .
$$

The superscript ${ }^{\circ}$ indicates that it is the previous time (according to the explicit scheme).

Equation (29) can be written as:

$$
\left(\Phi_{p}-\Phi_{p}^{\circ}\right) \frac{\Delta x \Delta y \Delta z}{\Delta t}+\left(L_{e}-L_{w}\right)+\left(L_{n}-L_{s}\right)+\left(L_{h}-L_{b}\right)=\bar{S} \Delta x \Delta y \Delta z
$$


where:

$L_{e}=L_{x e} \Delta y \Delta z, L_{w}=L_{x w} \Delta y \Delta z, L_{n}=L_{y n} \Delta x \Delta z, L_{s}=L_{y s} \Delta x \Delta z, L_{h}=L_{z h} \Delta x \Delta y$ and $L_{b}=L_{z b} \Delta x \Delta y$.

The source term $\bar{S}$ is linearized as:

$$
\bar{S}=S_{c}+S_{p} \Phi_{p}
$$

Notably, $S_{p}$ must be negative to provide stability for numerical simulations.

Integrating the continuity of Equation (9) written in a dimensionless form on to the same control volume, we obtained:

$$
\left(V_{x e} \Delta y \Delta z-V_{x w} \Delta y \Delta z\right)+\left(V_{y n} \Delta x \Delta z-V_{y s} \Delta x \Delta z\right)+\left(V_{z h} \Delta x \Delta y-V_{z b} \Delta x \Delta y\right)=0 .
$$

We considered:

$F_{e}=V_{x e} \Delta y \Delta z$ and $F_{w}=V_{x w} \Delta y \Delta z ; F_{n}=V_{y n} \Delta x \Delta z$ and $F_{s}=V_{y s} \Delta x \Delta z ; F_{h}=V_{z s} \Delta x \Delta y$ and $F_{b}=V_{z b} \Delta x \Delta y$

Equation (34) becomes:

$$
\left(F_{e}-F_{w}\right)+\left(F_{n}-F_{s}\right)+\left(F_{h}-F_{b}\right)=0
$$

where $F_{e}, F_{w}, F_{n}, F_{s}, F_{h}$ and $F_{b}$ represent the mass flow rates through the faces of the control volume.

Multiplying the discretized continuity Equation (36) by $\Phi_{p}$ and subtracting it from the general discretized Equation (31) then gives:

$$
\begin{aligned}
\Phi_{p}\left(\frac{\Delta x \Delta y \Delta z}{\Delta t}-S_{p} \Delta x \Delta y \Delta z\right)+ & \left(L_{e}-F_{e} \Phi_{p}\right)-\left(L_{w}-F_{w} \Phi_{p}\right)+\left(L_{n}-F_{n} \Phi_{p}\right)-\left(L_{s}-F_{s} \Phi_{p}\right)+\left(L_{h}-F_{h} \Phi_{p}\right) \\
& -\left(L_{b}-F_{b} \Phi_{p}\right)=\left(S_{c} \Delta x \Delta y \Delta z+\Phi_{p}^{\circ} \frac{\Delta x \Delta y \Delta z}{\Delta t}\right) .
\end{aligned}
$$

We considered the following expressions [21]:

$$
\begin{aligned}
& \left(L_{n}-F_{n} \Phi_{p}\right)=a_{N}\left(\Phi_{p}-\Phi_{N}\right),\left(L_{s}-F_{s} \Phi_{p}\right)=a_{S}\left(\Phi_{S}-\Phi_{p}\right),\left(L_{e}-F_{e} \Phi_{p}\right)=a_{E}\left(\Phi_{p}-\Phi_{E}\right), \\
& \left(L_{w}-F_{w} \Phi_{p}\right)=a_{W}\left(\Phi_{W}-\Phi_{p}\right),\left(L_{h}-F_{h} \Phi_{p}\right)=a_{H}\left(\Phi_{p}-\Phi_{H}\right),\left(L_{b}-F_{b} \Phi_{p}\right)=a_{B}\left(\Phi_{B}-\Phi_{p}\right) .
\end{aligned}
$$

Equation (37) becomes:

$$
a_{p} \Phi_{p}=a_{E} \Phi_{E}+a_{W} \Phi_{W}+a_{N} \Phi_{N}+a_{S} \Phi_{S}+a_{H} \Phi_{H}+a_{B} \Phi_{B}+b_{p}
$$

where:

$$
a_{p}=a_{E}+a_{W}+a_{N}+a_{S}+a_{H}+a_{B}+\frac{\Delta x \Delta y \Delta z}{\Delta t}-S_{p} \Delta x \Delta y \Delta z \text { and } b_{p}=S_{c} \Delta x \Delta y \Delta z+\Phi_{p}^{\circ} \frac{\Delta x \Delta y \Delta z}{\Delta t} \text {. }
$$

By substituting the function $\Phi_{p}$ with the temperature $T$ in the general discretized Equation (39), we obtained the discretized energy equation:

$$
a_{p} T_{p}=a_{E} T_{E}+a_{W} T_{W}+a_{N} T_{N}+a_{S} T_{S}+a_{H} T_{H}+a_{B} T_{B}+b_{p} \text { with } b_{p}=T_{p}^{\circ} \frac{\Delta x \Delta y \Delta z}{\Delta t}
$$

By substituting the function $\Phi_{p}$ with the concentration $C$ in the general discretized Equation (39), we obtained the discretized mass equation:

$$
a_{p} C_{p}=a_{E} C_{E}+a_{W} C_{W}+a_{N} C_{N}+a_{S} C_{S}+a_{H} C_{H}+a_{B} C_{B}+b_{p} \text { with } b_{p}=C_{p}^{\circ} \frac{\Delta x \Delta y \Delta z}{\Delta t} .
$$

To develop the vorticity equation, we replaced the function $\Phi_{p}$ for each component $\omega_{x}, \omega_{y}$ and $\omega_{z}$, respectively, and obtained the following equations: 
- $\quad$ According to $x$-direction, $\Phi_{p}=\omega_{x}$ :

$$
a_{p} \omega_{x p}=a_{E} \omega_{x E}+a_{W} \omega_{x W}+a_{N} \omega_{x N}+a_{S} \omega_{x S}+a_{H} \omega_{x H}+a_{B} \omega_{x B}+b_{p}
$$

with

$$
\begin{aligned}
b_{p}=\omega_{x p}^{\circ} \frac{\Delta x \Delta y \Delta z}{\Delta t}+ & \omega_{x p}\left(\frac{V_{x E}-V_{x w}}{2}\right) \Delta y \Delta z+\omega_{y p}\left(\frac{V_{x S}-V_{x N}}{2}\right) \Delta x \Delta z+\omega_{z p}\left(\frac{V_{x H}-V_{x B}}{2}\right) \Delta x \Delta y \\
& +\operatorname{RaPr}\left(\frac{T_{H}-T_{B}}{2}\right) \Delta x \Delta y-\operatorname{RaPrN}\left(\frac{C_{H}-C_{B}}{2}\right) \Delta x \Delta y .
\end{aligned}
$$

- According to $y$-direction, $\Phi_{p}=\omega_{y}$ :

$$
a_{p} \omega_{x p}=a_{E} \omega_{y E}+a_{W} \omega_{y W}+a_{N} \omega_{y N}+a_{S} \omega_{y S}+a_{H} \omega_{y H}+a_{B} \omega_{y B}+b_{p}
$$

with

$$
b_{p}=\omega_{y p}^{\circ} \frac{\Delta x \Delta y \Delta z}{\Delta t}+\omega_{y p}\left(\frac{V_{y E}-V_{y w}}{2}\right) \Delta y \Delta z+\omega_{y p}\left(\frac{V_{y S}-V_{y N}}{2}\right) \Delta x \Delta z+\omega_{z p}\left(\frac{V_{y H}-V_{y B}}{2}\right) \Delta x \Delta y
$$

- $\quad$ According to $z$-direction, $\Phi_{p}=\omega_{z}$ :

$$
a_{p} \omega_{z p}=a_{E} \omega_{z E}+a_{W} \omega_{z W}+a_{N} \omega_{z N}+a_{S} \omega_{z S}+a_{H} \omega_{z H}+a_{B} \omega_{z B}+b_{p}
$$

with

$$
\begin{gathered}
b_{p}=\omega_{z p}^{\circ} \frac{\Delta x \Delta y \Delta z}{\Delta t}+\omega_{z p}\left(\frac{V_{z E}-V_{z w}}{2}\right) \Delta y \Delta z+\omega_{z p}\left(\frac{V_{z S}-V_{z N}}{2}\right) \Delta x \Delta z+\omega_{z p}\left(\frac{V_{z H}-V_{z B}}{2}\right) \Delta x \Delta y \\
-\operatorname{RaPr}\left(\frac{T_{E}-T_{W}}{2}\right) \Delta y \Delta z+\operatorname{RaPrN}\left(\frac{C_{E}-C_{W}}{2}\right) \Delta y \Delta z .
\end{gathered}
$$

The potential vector Equation (10) was discretized using centered differences, and three equations were obtained for each component: $x, y$ and $z$.

$$
\begin{aligned}
& \Psi_{x p}=\frac{1}{2\left(\frac{1}{\Delta x^{2}}+\frac{1}{\Delta y^{2}}+\frac{1}{\Delta z^{2}}\right)}\left(\frac{\Psi_{x E}+\Psi_{x W}}{\Delta x^{2}}+\frac{\Psi_{x N}+\Psi_{x S}}{\Delta y^{2}}+\frac{\Psi_{x H}+\Psi_{x B}}{\Delta z^{2}}+\omega_{x p}\right) \\
& \Psi_{y p}=\frac{1}{2\left(\frac{1}{\Delta x^{2}}+\frac{1}{\Delta y^{2}}+\frac{1}{\Delta z^{2}}\right)}\left(\frac{\Psi_{y E}+\Psi_{y W}}{\Delta x^{2}}+\frac{\Psi_{y N}+\Psi_{y S}}{\Delta y^{2}}+\frac{\Psi_{y H}+\Psi_{y B}}{\Delta z^{2}}+\omega_{y p}\right) \\
& \Psi_{z p}=\frac{1}{2\left(\frac{1}{\Delta x^{2}}+\frac{1}{\Delta y^{2}}+\frac{1}{\Delta z^{2}}\right)}\left(\frac{\Psi_{z E}+\Psi_{z W}}{\Delta x^{2}}+\frac{\Psi_{z N}+\Psi_{z S}}{\Delta y^{2}}+\frac{\Psi_{z H}+\Psi_{z B}}{\Delta z^{2}}+\omega_{z p}\right)
\end{aligned}
$$

\subsection{Algorithm Solving Steps}

The equations system obtained was solved by an iterative method with successive relaxations [22]. The power law scheme was used to treat the convection-diffusion terms and the time step was taken to $10^{-4}$.

A numerical code was developed using the FORTRAN programming language. In this numerical code, the following steps were followed:

1. Initialization;

2. Solving the Energy equation;

3. Solving the mass equation

4. Solving the vorticity equation and calculating the boundary vorticity;

5. Solving the velocity potential-vector equation.

Steps 2 to 5 were repeated until a convergence criterion was satisfied. The solution was considered to be acceptable when the maximum residuals of the mass, momentum, energy and diffusion equations of the control volume of the network are less than $10^{-5}$. 
The following convergence criterion was achieved for each time step and for each dependent variable $(\Delta)$ :

$$
\frac{\max \left|\Delta^{m}-\Delta^{m-1}\right|}{\max \left|\Delta^{m}\right|} \leq 10^{-5} \text {, where the exponent }(\mathrm{m}) \text { refers to the temporal iteration. }
$$

\subsection{Grid Dependency Study and Code Verification}

A grid sensitivity test was conducted (Table 2) for $N=0$ and $R a=5 \times 10^{4}$. Four distinct grid sizes were investigated $\left(41^{3}, 51^{3}, 61^{3}\right.$ and $\left.71^{3}\right)$. The average Nusselt number was taken as the sensitive variable. The incremental rise in the average Nusselt number from grid size $61^{3}$ to grid size $71^{3}$ was approximately $0.15 \%$. Thus, considering the computing efficiency and reliability, a grid size of $61^{3}$ was retained.

Table 2. Grid sensitivity test for reference case (a), $N=0$ and $R a=5 \times 10^{4}$.

\begin{tabular}{cccc}
\hline Grid Size & $\overline{\boldsymbol{N}} \boldsymbol{u}$ & Percentage Increase & Incremental Increase \\
\hline $41^{3}$ & 2.8199911 & - & - \\
$51^{3}$ & 2.9932441 & 6.143743 & - \\
$61^{3}$ & 3.0344111 & 7.60357 & 1.459827 \\
$71^{3}$ & 3.0385622 & 7.750773 & 0.147203 \\
\hline
\end{tabular}

The numerical code was then verified by comparing the results with those obtained in two different simulation cases: the first case concerns a three-dimensional cavity with a horizontal gradient of temperature and concentration filled with an aqueous solution $\operatorname{Pr}=10, L e=10$ and $R a=10^{5}$ developed by Sezai and Mohamad [23], and the second one is related to a square cavity of dimension $L$ filled with nanofluids containing a square hot obstacle centered in the cavity with a width $\mathrm{W}$. The Prandtl number is equal to 6.2 and the Rayleigh number is equal to $10^{6}$. The nanofluid is water-Cu with a fraction of 0.04 [24] (Table 3). It is clear that the results of our code are in good agreement with those proposed by Rahmati and Tahery [24]. Indeed, the maximum deviation is of the order of $1.8 \%$. The flow structure, iso-concentration and iso-temperature obtained by our code are similar to those obtained by Sezai and Mohamad [23] in the two planes $z=0.5$ and $x=0.5$ (Figure 3).

Table 3. Comparison of the obtained average Nusselt number with the results of Rahmati et al. [24] at $R a=10^{6}$.

\begin{tabular}{cccc}
\hline W/L & $\begin{array}{c}\text { Nusselt Average, } \\
\text { Rahmati et al. Works }\end{array}$ & $\begin{array}{c}\text { Nusselt Average, } \\
\text { Present Works }\end{array}$ & Error \% \\
\hline 0.2 & 13 & 12.883 & 0.9 \\
0.3 & 13.5 & 13.316 & 1.36 \\
0.4 & 14 & 13.732 & 1.91 \\
0.5 & 14.25 & 13.974 & 1.93 \\
0.6 & 13.8 & 13.675 & 0.91 \\
\hline
\end{tabular}



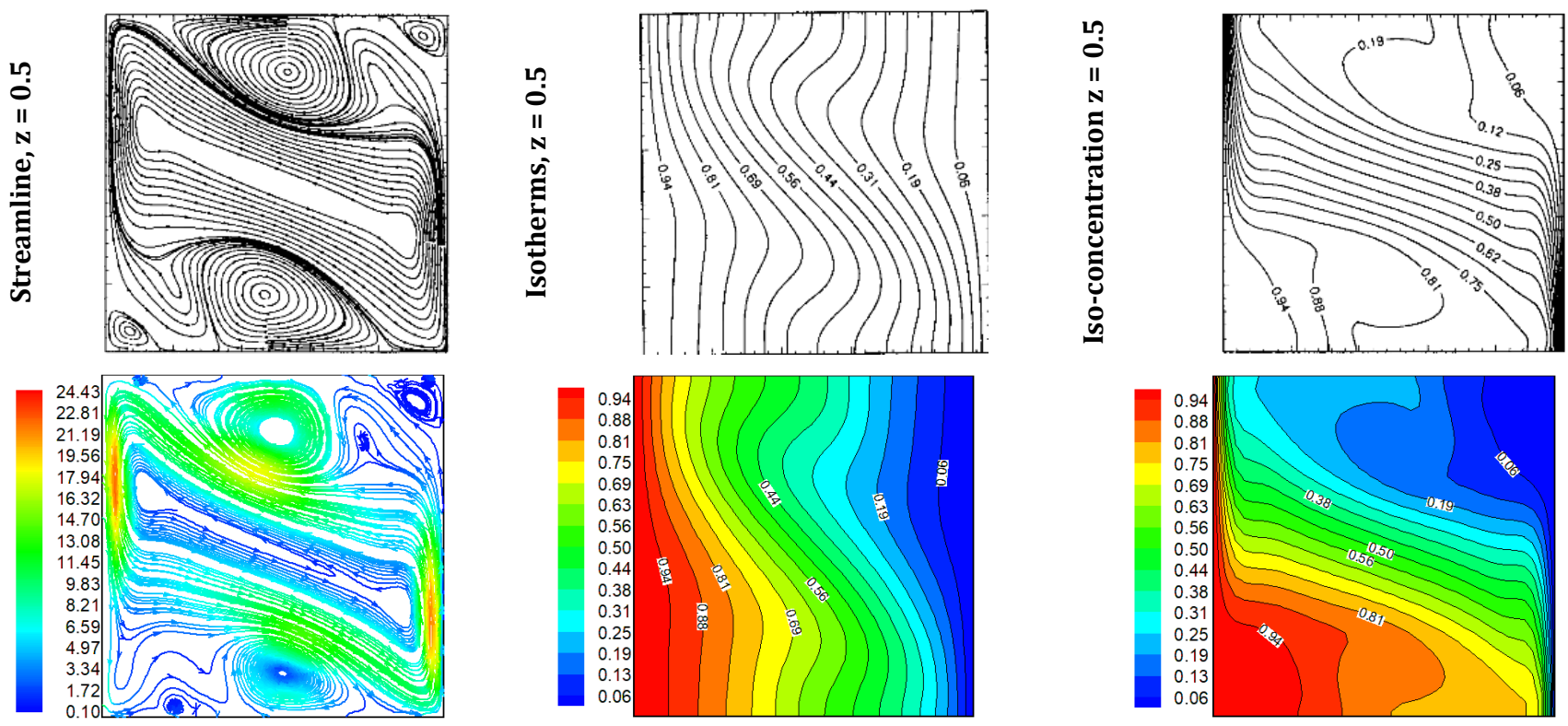

(a): mid plane, $\mathrm{X}-\mathrm{Y}$ at $\mathrm{z}=0.5$
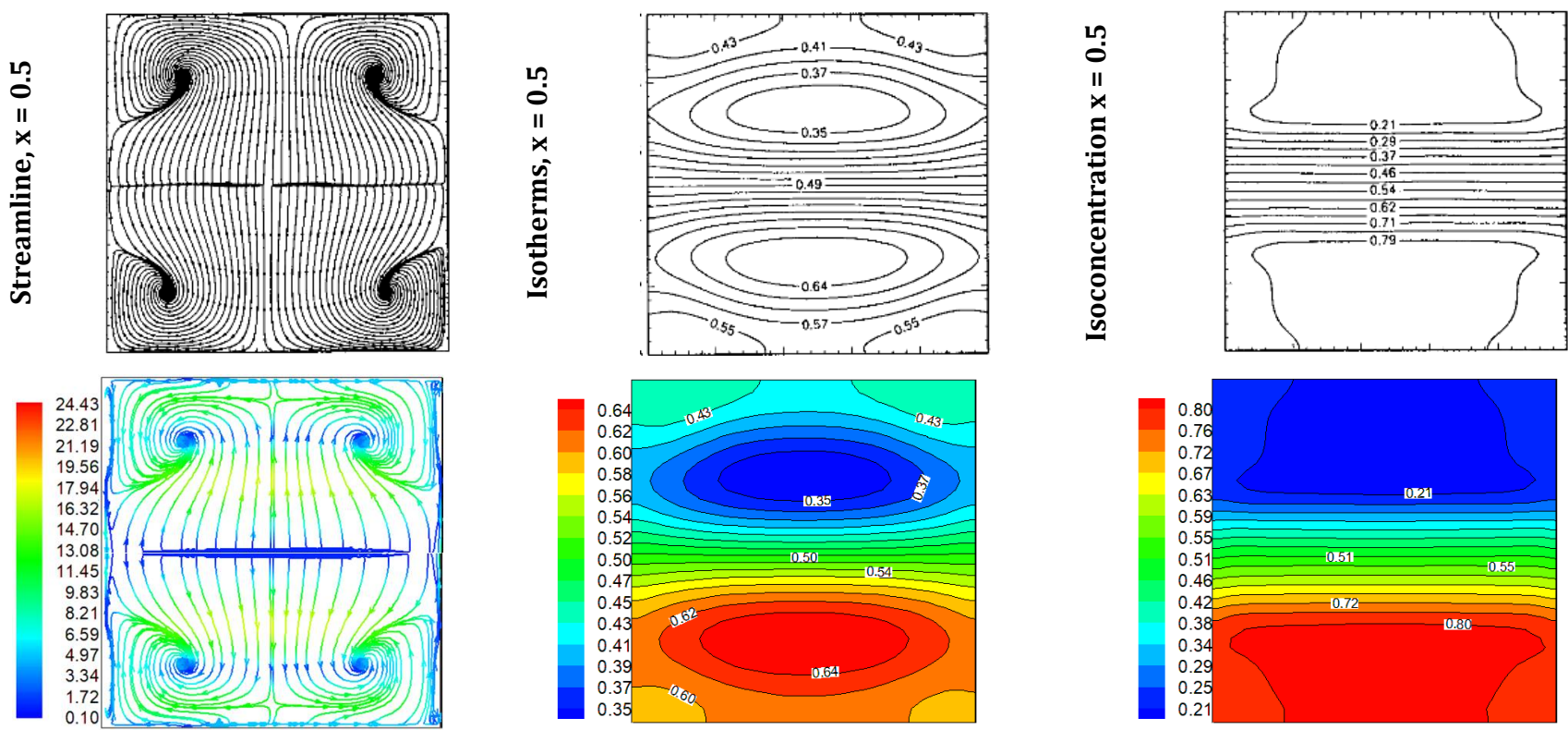

(b): mid plane, $\mathrm{Y}-\mathrm{Z}$ at $\mathrm{x}=0.5$

Figure 3. Comparison of the present results (bottom) of Streamlines, iso-temperature and iso-concentration with the results of Sezai and Mohamad [23] (top) at $R a=10^{5}, \operatorname{Pr}=10$ and $L e=10,(\mathbf{a})$ : X-Y-mid-plane, $(z=0.5)$ and (b): Y-Z-mid-plane $(x=0.5)$.

\section{Results and Discussion}

The results presented in this paper were obtained for ranges of governing parameters: Rayleigh numbers $\left(10^{3}\right.$ to $\left.10^{5}\right)$, buoyancy ( -2 to 2 ) and a barrier height ( 0.1 to 0.75$)$. The Prandtl number was fixed at $\operatorname{Pr}=0.7$ and the Lewis number was $L e=1.2$. Iso-concentration, iso-temperature and particle trajectories are presented to illustrate the dispersion of the contaminant, the effectiveness of the barrier and the heat and mass transfer in the building. Interesting results were seen for fluid flow and heat and mass transfer through the cavity, depending on the chosen values of the governing parameters, the sign of the buoyancy ratio and the height of the barrier. 


\subsection{Flow Patterns in Reference Case}

The flow patterns in the reference case are studied in this subsection. The reference case is the case involving the building without a barrier.

Figure 4 presents the particles trajectories, iso-concentrations and iso-temperatures at $N=0$ for $R a=10^{3}$ and $10^{5}$. The flow structure is characterized by a single clockwise rotating vortex. The contaminant particles move from the part at high concentration in the right bottom wall to the whole of the cavity due to the buoyancy forces. The increase of Rayleigh numbers increases the magnitude of fluid velocity without tangible variation of the flow structure. The distribution of iso concentration shows a diffusive dispersion on contaminant when $R a=10^{3}$ is localized in the right side of the cavity due to the high concentration of the contaminant in the right building floor. The dispersion of the contaminant becomes convective when $R a=10^{5}$. The dispersion of the contaminant is mainly directed upwards at the corners of the building and to the left in the core of the building. The iso-temperature structure shows that the conductive mode is dominant in case of $R a=10^{3}$. When $R a=10^{5}$, the thermal gradient increases and the convective mode becomes dominant.

Ra

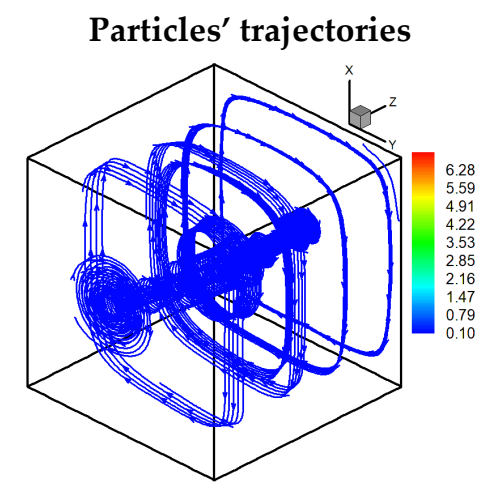

$10^{5}$

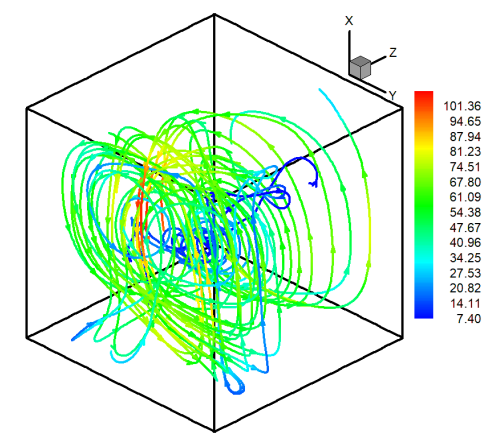

Iso-concentration
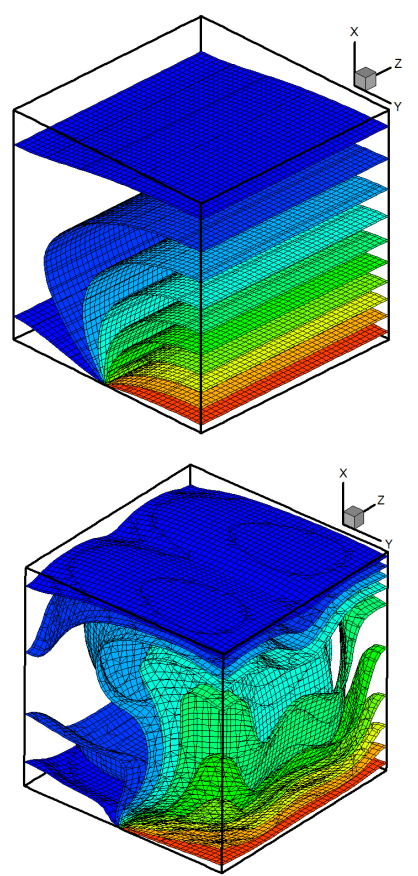

Iso-temperature
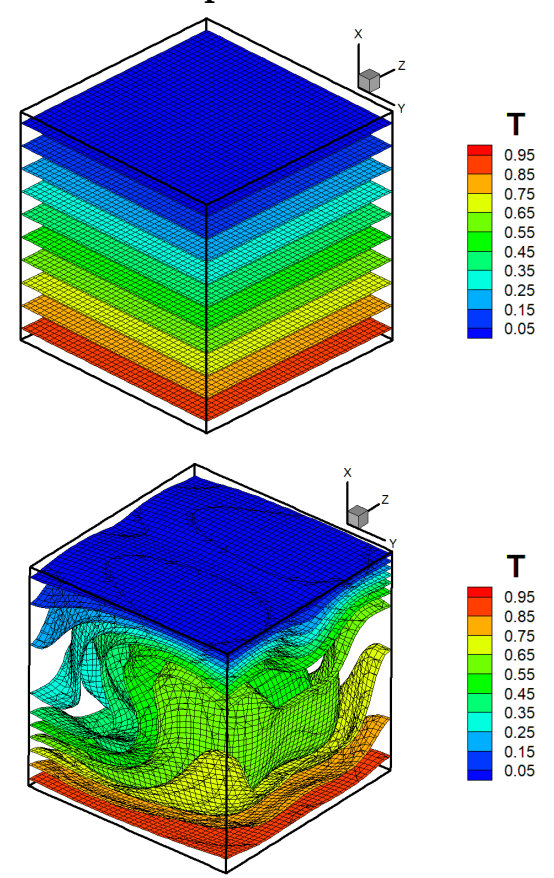

Figure 4. Particles' trajectories, iso-concentration and iso-temperature in the building without a barrier for $N=0, R a=10^{3}$ and $10^{5}$.

5.2. Effect of Rayleigh, Barrier Height and Boyancy Ratio on the Dispersion of the Contaminant 5.2.1. Effect of Rayleigh and Barrier Height for $N=0$

Figures 5-7 presents the effect of barrier height on the contaminant flow dispersion, iso-concentrations and the iso-temperatures, respectively, for different Rayleigh numbers and $N=0$. When the barrier is placed in the base wall of the cavity, the flow of fluid in the area to the right of the barrier is governed by thermal and solutal buoyancy forces. However, in the area to the left of the barrier, only thermal buoyancy forces govern the flow. Two heights of the barrier were studied in this work. The first height is 0.25 of the height of the cavity and the second is 0.5 . 
Ra

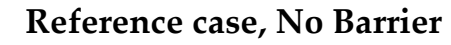

$10^{3}$

$10^{4}$

$5 \times 10^{4}$

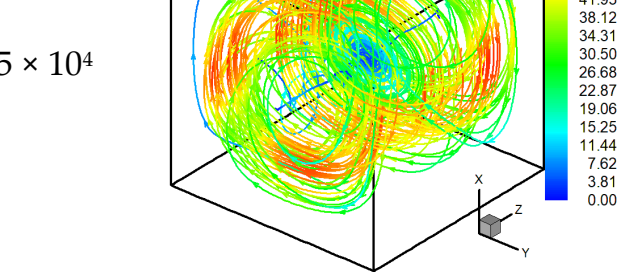

With Barrier, $\mathrm{H}_{1}=0.25$
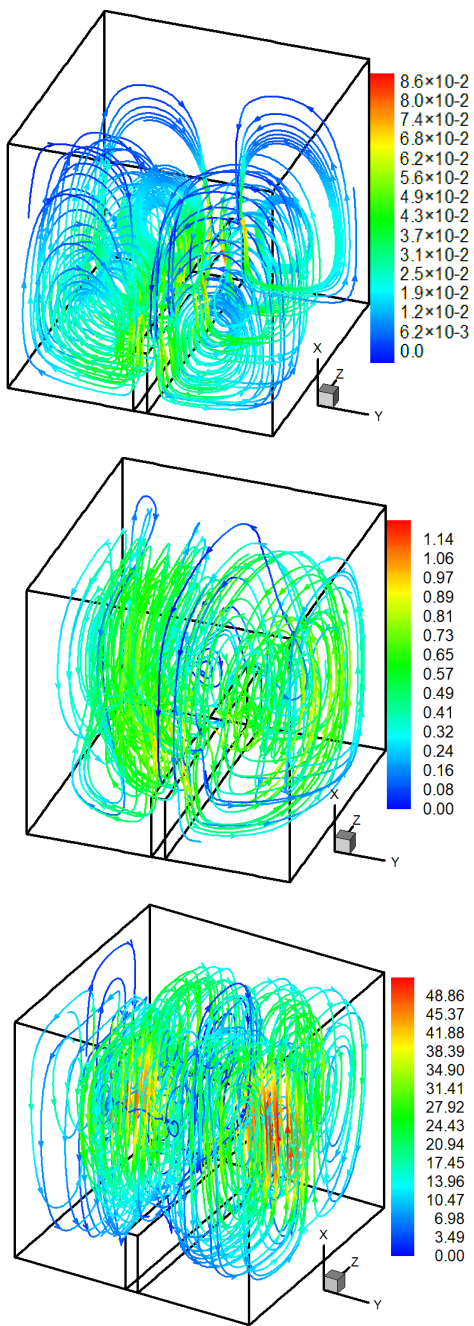

With Barrier, $\mathrm{H}_{2}=0.5$
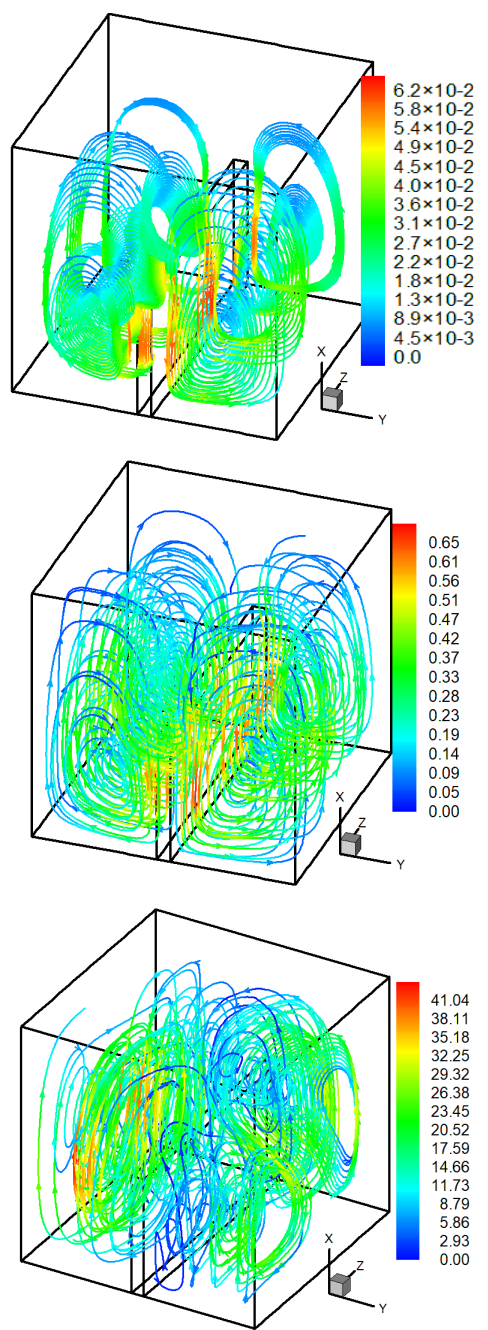

Figure 5. Particles' trajectories of the fluid in the building for different $R a$, effect of two barriers' heights for $N=0$.

Figure 5 shows the effect of Rayleigh numbers on the flow structure in the building in three cases when $N=0$; the first case represented in the first column is the barrier-free case (the reference case), the second case with the first height (0.25) whose results are presented in the middle column and the third case with the second height (0.5) in the third column. When $R a=10^{3}$, it can be noticed that the presence of the barrier limited the dispersal of the contaminant throughout the cavity and that the magnitude of the velocity decreased remarkably compared to the case without the barrier (the reference case). It is also noticed that parallel flow rollers in the X-Y plane appeared when the barrier is installed. Additionally, at this Ra number, the effect of the barrier height cannot observed at the flow structure. However, a slight decrease in velocity can be observed when the barrier height is at its maximum. For $R a=10^{4}$, there is a noticeable change in the flow structure for the first obstacle height, and the flow structure is characterized by parallel rotating vortices in the $\mathrm{X}-\mathrm{Z}$ plane. The flow structure is almost similar on both sides of the obstacle with slight differences, which is represented by the presence of small vortices positioned at the bottom in the right side of the cavity. On the other hand, for the second height of the obstacle, the flow structure is not changed, and only a slight increase in the velocity can be noticed. 
Ra

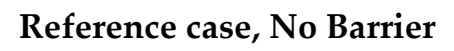

$10^{3}$

$10^{4}$
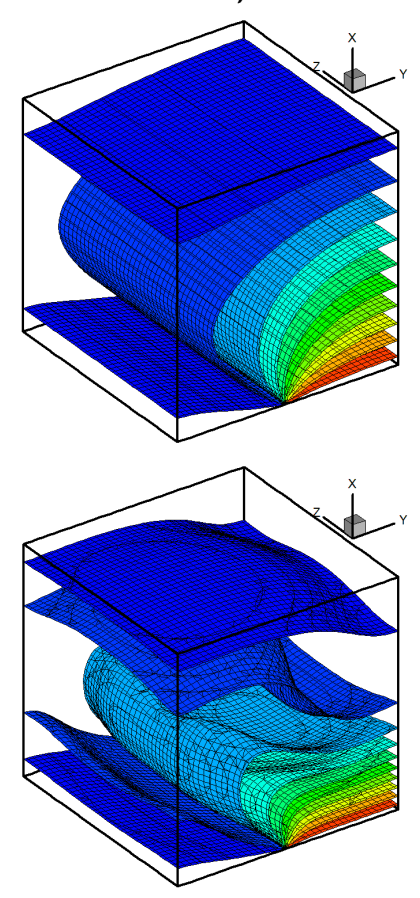

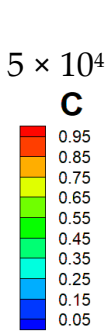

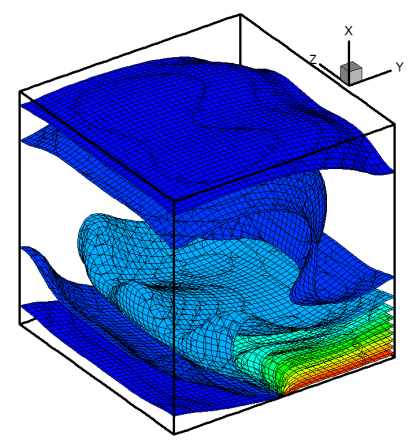

With Barrier, $\mathrm{H}_{1}=0.25$
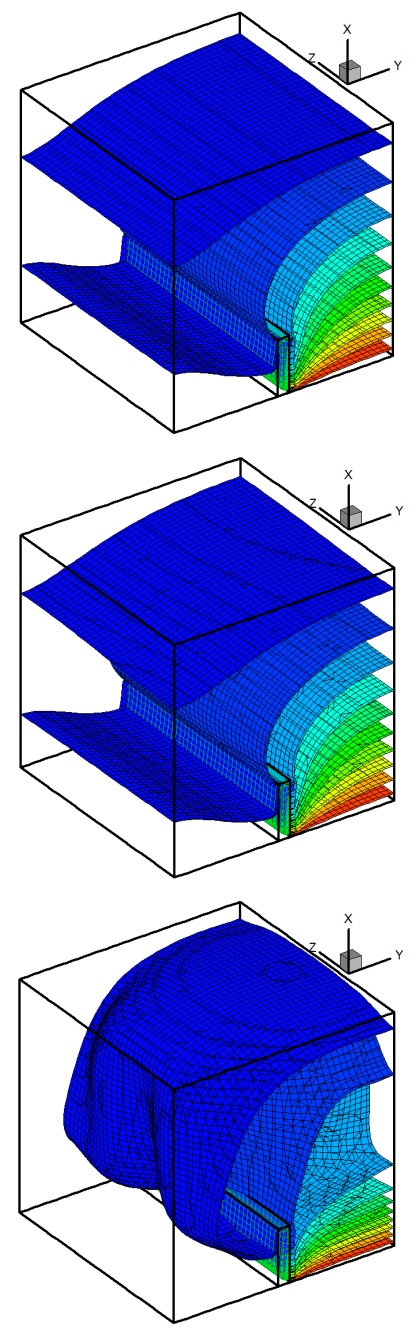

With Barrier, $\mathrm{H}_{2}=0.5$
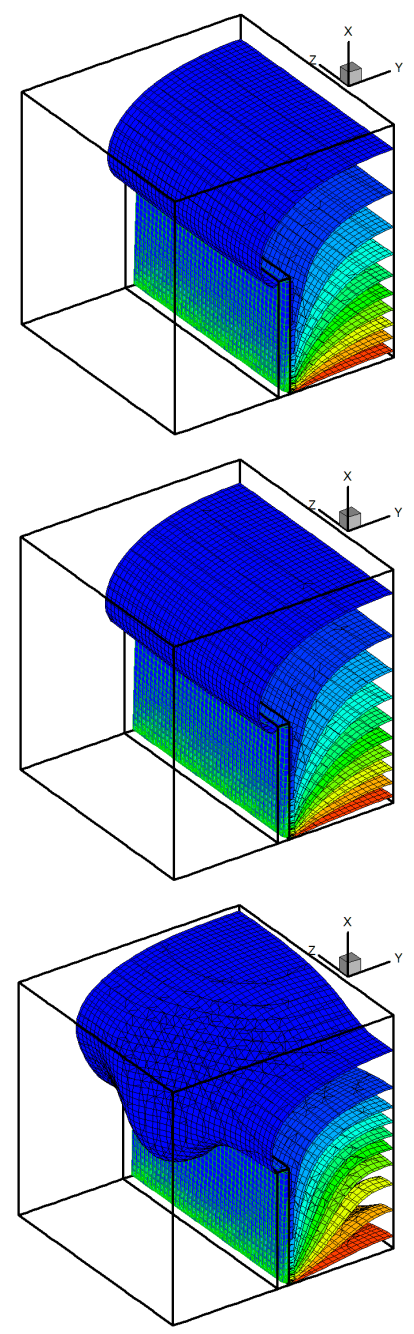

Figure 6. Contaminant iso-surfaces of concentration for different $R a$, effect of two barriers' heights for $N=0$.

When $R a=5 \times 10^{4}$, it can be observed that the velocity of the flow is further increased and in the absence of the barrier, the contaminant is dispersed in the whole cavity. Thus, the main flow always comprises of a single central vortex that encloses the entire volume with a spiral flow. The increase in the Rayleigh number for the case of the first barrier height induced a second change in the flow structure compared to the $R a=10^{4}$. In this case, the flow pattern is recognized by two vortices rotating in opposite directions in the $\mathrm{X}-\mathrm{Z}$ plane. The flow structure is similar on both sides of the barrier. The increase in the Rayleigh number in the cavity with the second height of the barrier also induced a change in the flow structure compared to $R a=10^{3}$ and $R a=10^{4}$. In this case too, the flow structure is characterized by two counter-rotating vortices in the $X-Z$ plane on the right side of the barrier, while it is a single vortex on the left side. Such differences in the flow structure between the two sides of the barrier may be related to the combined effects of the thermal and solutal volume forces on the right side compared to the single thermal effect on the left side. 
Ra

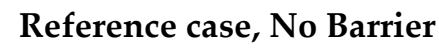

$10^{3}$

$10^{4}$
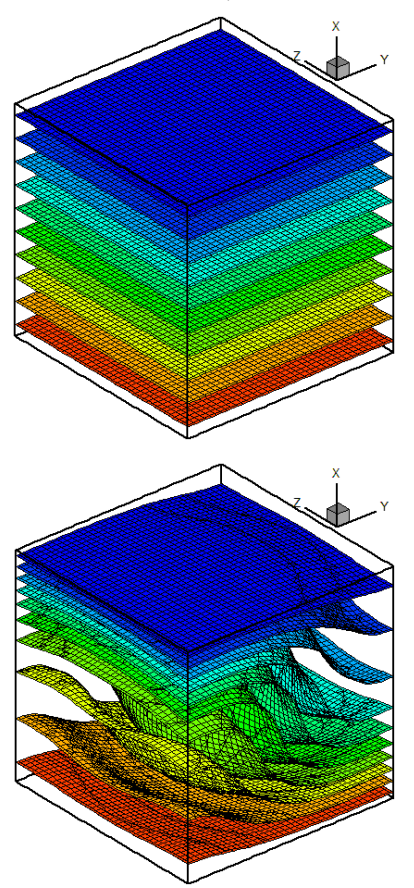

$5 \times 10^{4}$
$\mathbf{T}$
$\mathbf{T}$
0.95
0.85
0.75
0.65
0.55
0.55
0.45
0.25
0.15
0.15
0.05
With Barrier, $\mathrm{H}_{1}=0.25$
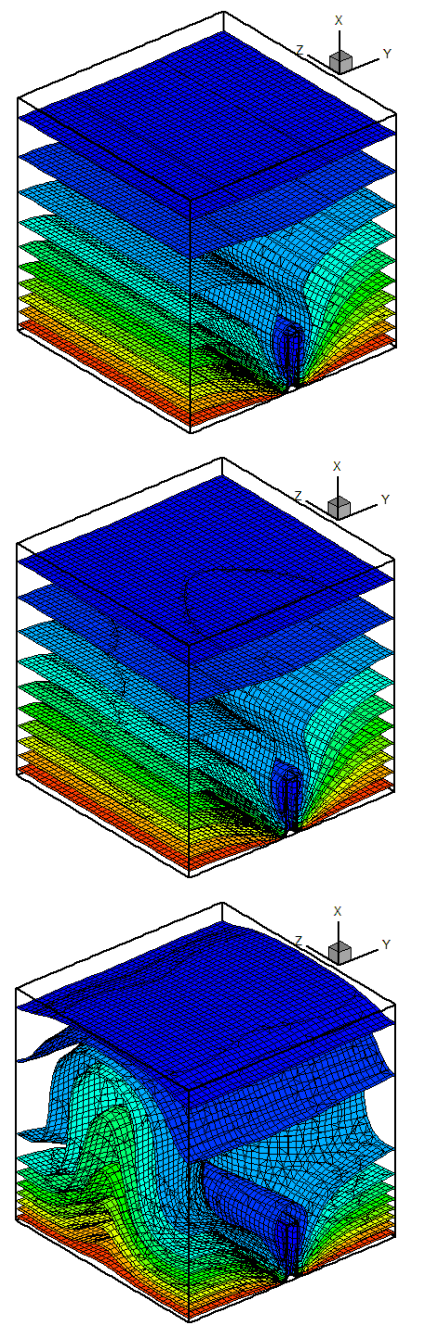

With Barrier, $\mathrm{H}_{2}=0.5$
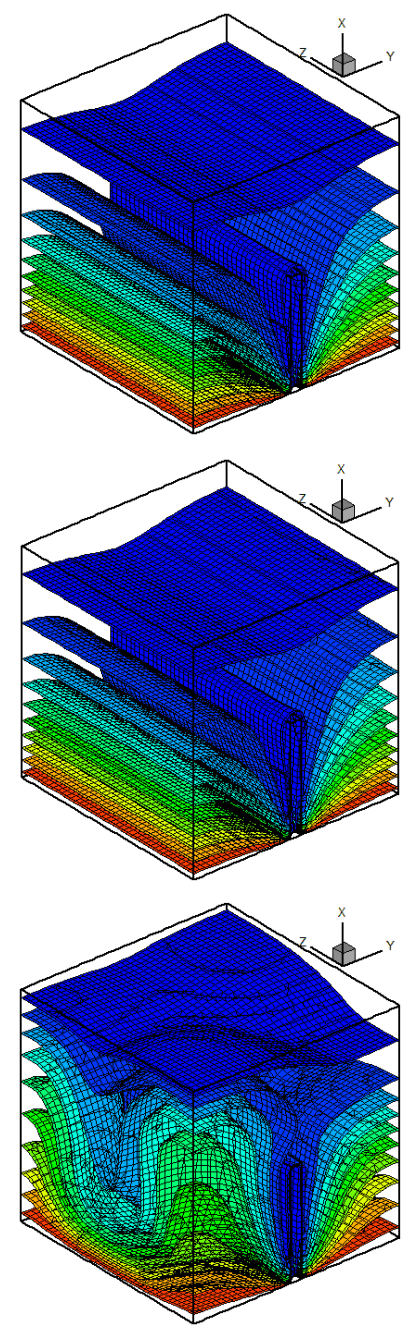

Figure 7. Temperature iso-surfaces in the building for different $R a$, effect of two barriers' heights for $N=0$.

Figure 6 illustrates the effect of Rayleigh numbers on the iso-surfaces of contaminant concentration in the building in the three cases studied. When $R a=10^{3}$, the concentration iso-surfaces are distributed from the contaminant emitting area. They are parallel and located in the right part of the cavity. The solutal gradient is low and the diffusive regime is dominant. The diffusion of the contaminant is mainly directed towards the top of the cavity by migration under the influence of the side walls. For Rayleigh numbers equal $10^{4}$ and $5 \times 10^{4}$, on the one hand an increase in the solutal gradient can be noticed while on the other hand a convective effect appears. The contaminant starts to disperse horizontally towards the left part of the cavity and contaminates the remaining parts of the cavity too. The implementation of the barrier shows an effect limiting the horizontal dispersion of the contaminant. For both Rayleigh numbers i.e., $10^{4}$ and $5 \times 10^{4}$, it can be concluded that the second height strongly opposes the dispersion of the contaminant. The convective effect is attenuated by the presence of the barrier and pushes the contaminant to only migrate vertically near the side walls.

Figure 7 shows the effect of Rayleigh numbers on temperature iso-surfaces. In the absence of a barrier and when $R a=10^{3}$, it can be noticed that the temperature iso-surfaces are parallel and that the conductive regime is dominant. The thermal convective regime appears from the value of $R a=10^{4}$, as evidenced by the distribution of the temperature iso-surfaces that start to deform from the side in the cavity that has a higher thermal gradient with the source of contamination. For $R a=5 \times 10^{4}$, the temperature iso-surfaces 
form an S-shape, indicating the complete development of the thermal convective mode. In the presence of the barrier and for both Rayleigh numbers $10^{3}$ and $10^{4}$, it can be noticed that the distribution of temperature iso-surfaces is similar and characterized by a dominant conductive thermal regime. Also, the thermal gradient is weak on both sides of the barrier. When $R a=5 \times 10^{4}$, complete development of the thermally convective mode can be seen It can further be observed that the structure of the temperature iso-surfaces depends on the height of the barrier. Indeed, for the first height of the barrier, the thermal gradients are high at the corners of the cavity and low in the middle part. However, for the second height, it can be noticed that they are low at the corners and high in the middle.

\subsubsection{Effect of Rayleigh and Barrier Height for $N=-0.2$}

The nature of the contaminant and its characteristics have a major effect on the manner of its dispersal. For the case where the contaminant is in a liquid sprayed phase loaded with viruses or bacteria and is bound to its environment during its emission and dispersion, the dispersion of this existing viral load in the pulverized liquid phase is then governed by gravity having a descending effect, which is opposed to the effect of natural convection. In this case, the effects of the forces of solutal and thermal volumes are then opposed. This situation can be modeled with the negative buoyancy ratio.

Figure 8 presents the effect of Rayleigh numbers on the flow structure represented by particles' trajectories in the building in the three cases of this study when $N=-0.2$. For all Rayleigh numbers, the flow structure in the reference case is characterized by a single vortex in the $\mathrm{X}-\mathrm{Y}$ plane rotating in a clockwise direction. Increasing the Rayleigh number increases the flow velocity. When the building is equipped with the barrier, it is noticed that the variation of the Rayleigh number has a double effect on the flow structure as well as on the flow velocity.

When $R a=10^{4}$, the flow structure is similar in both cases, as shown by barrier height 1 and 2. Indeed, the flow is then characterized by two vortexes rotating in opposite directions: one on left of the barrier and the other on the right. The one on the right, where the contaminant's emitting surface is, the vortex rotates in a counterclockwise direction. On the other hand, the vortex on the left rotates in a clockwise direction. The increase in the Rayleigh number to $1.5 \times 10^{4}$ causes a change in the flow structure in the case of height 1 of the barrier, and this flow structure remains unchanged in the case of height 2 . The flow structure is characterized by a vortex rotating in the core of the $\mathrm{X}-\mathrm{Z}$ plane, turning counterclockwise. It is from $R a=2 \times 10^{4}$ that the flow structure in the second height of the barrier has this new structure. It can also be noticed that the magnitude of the particle velocity is lower on the right side of the barrier, on the side of the contaminant emitting surface when compared to the left side.

When $R a=5 \times 10^{4}$, the flow structure changes again in both cases and becomes characterized by two oppositely rotating vortexes carried by rollers in the $\mathrm{Y}-\mathrm{Z}$ plane along the entire cavity. It can be noticed that the intensity of the velocity is lower on the side of the contaminant emitting surface compared to the region to the left of the barrier. This result can be explained by the competition between the thermal and solutal volume forces in the zone on the right side of the barrier, as the negative buoyancy ratio. In contrast, only the thermal volume forces govern the flow in the zone on the left side of the barrier. When Rayleigh number is equal to $10^{5}$, it can be noticed that the flow structure for the first case becomes multicellular. The flow velocity has increased in both cases and the maximum velocities are observed in the zone to the left of the barrier where only the forces of thermal volumes govern the flow. 
Ra

$$
\text { Reference case, No Barrier }
$$

$10^{4}$

$1.5 \times 10^{4}$

$2 \times 10^{4}$

$5 \times 10^{4}$

$10^{5}$
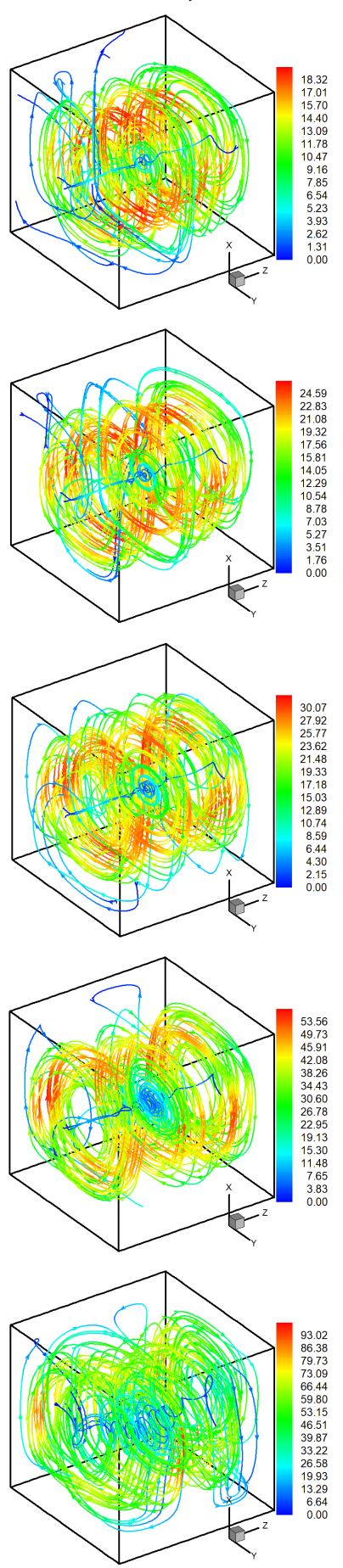

With Barrier, $\mathrm{H}_{1}=0.25$
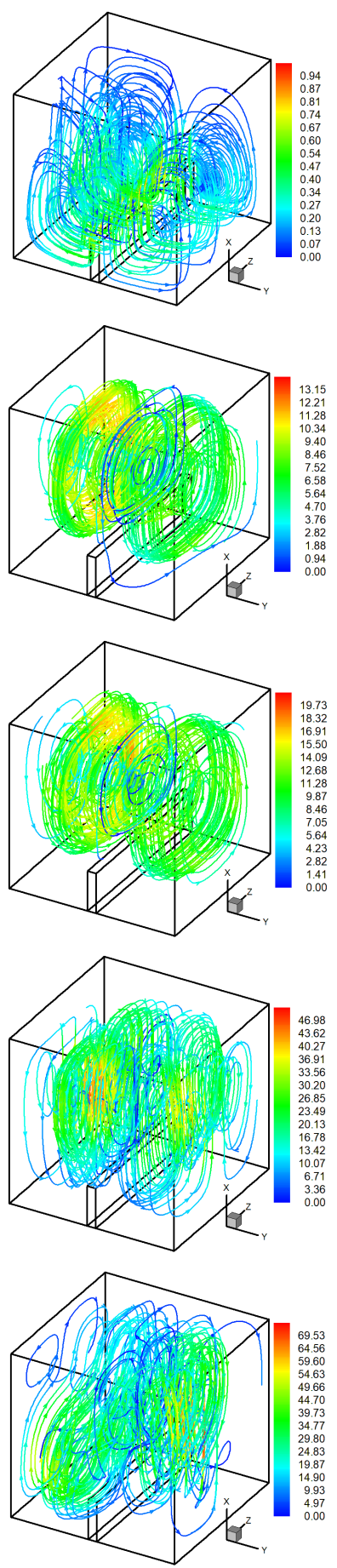

With Barrier, $\mathrm{H}_{2}=0.5$
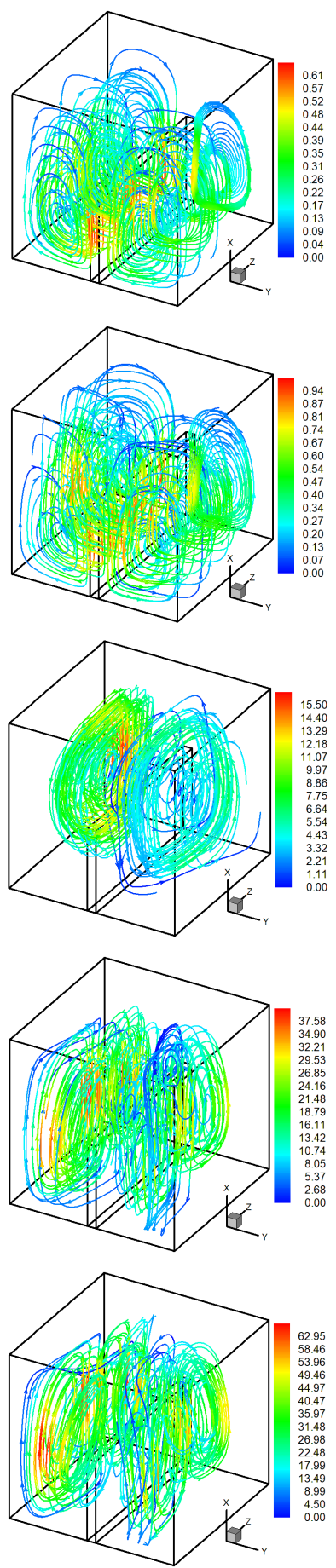

Figure 8. Particles' trajectories of the fluid in the building for different $R a$, effect of two barriers' heights for $N=-0.2$.

Figure 9 presents the effect of Rayleigh numbers for $N=-0.2$ on the iso-surface of contaminant concentration in the building for the three cases in this study. The increase of the Rayleigh number in the reference case, the building without barriers, increases the concentration gradient close to the contaminant emitting surface. The structure of the iso-surfaces shows that the solutal convective effect is predominant. The increase of the Rayleigh number also increases the dispersal effect of the contaminant mainly in a horizontal way at the bottom of the building. It can be noticed that the placement of the barrier with height 1 reduces the dispersion of the contaminant towards the left zone. For 
the low Rayleigh number, the dispersion is horizontal, under the effect of diffusion, which is the dominant regime. For $R a=5 \times 10^{4}$, it can be noticed that the dispersion of the contaminant becomes governed by the solutal convection. It can be noticed that the second height of the barrier is more effective in terms of limiting the dispersion of the contaminant towards the left zone. However, it can be observed that this case has led to an increase in the vertical dispersion of the contaminant compared to the other studied cases.

Ra

$10^{4}$

$1.5 \times 10^{4}$

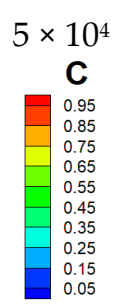

\section{Reference case, No Barrier}
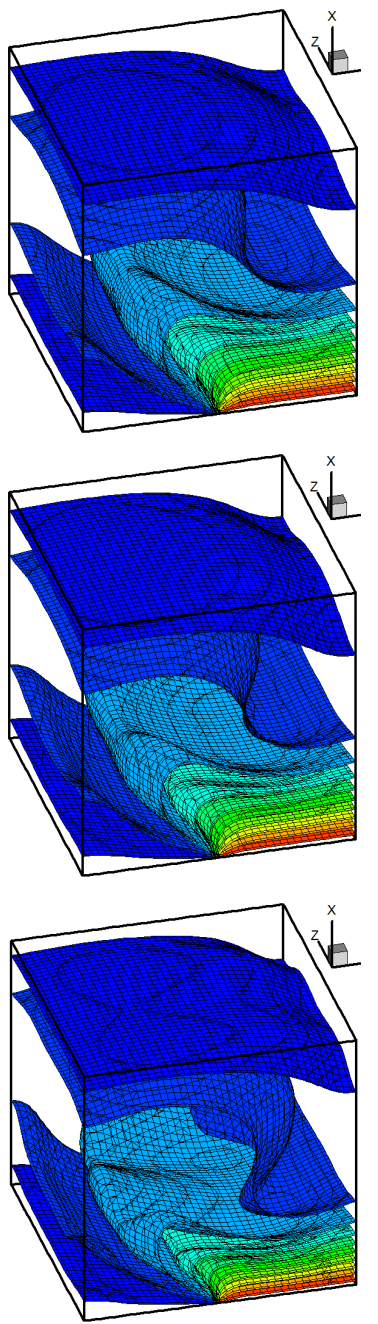

With Barrier, $\mathrm{H}_{1}=0.25$
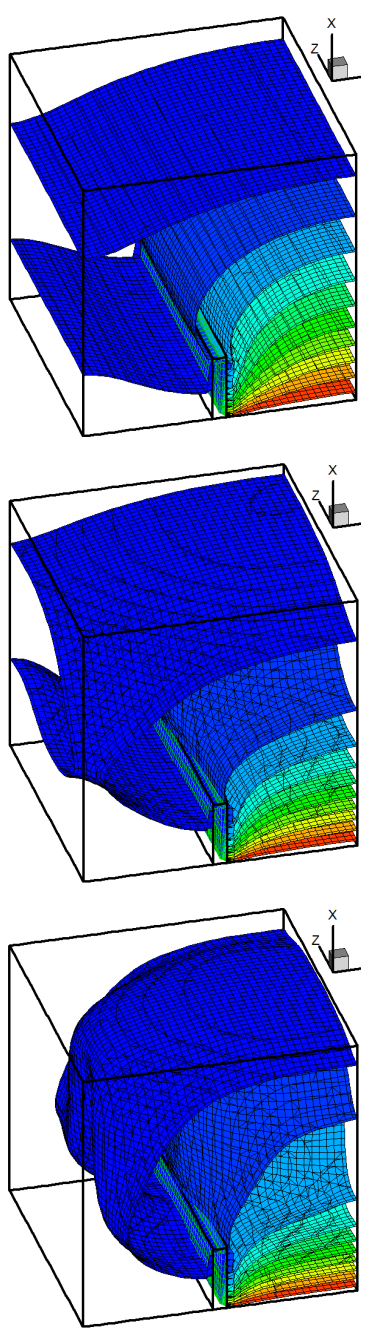

With Barrier, $\mathrm{H}_{2}=0.5$
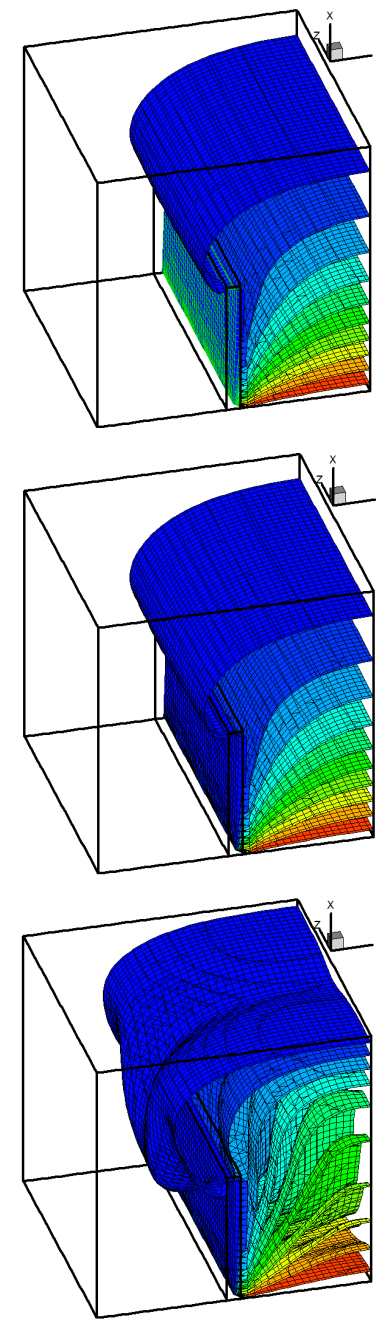

Figure 9. Contaminant iso-surfaces of concentration for different $R a$, effect of two barriers' heights for $N=-0.2$.

Figure 10 shows the effect of Rayleigh numbers on temperature iso-surfaces. In the absence of a barrier, the thermal convective regime appears from the value of $R a=10^{4}$, while increasing the Rayleigh number increases the thermal gradient. In the presence of barrier height 1 , the convective regime appears from Rayleigh $1.5 \times 10^{4}$, while for barrier height 2 , the convective regime is observed for $R a=5 \times 10^{4}$. 
Ra

Reference case, No Barrier

$10^{4}$

$1.5 \times 10^{4}$

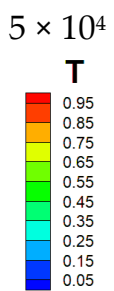

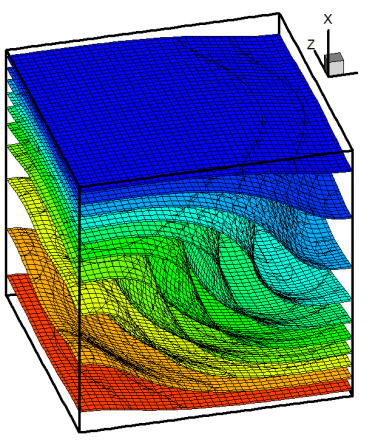
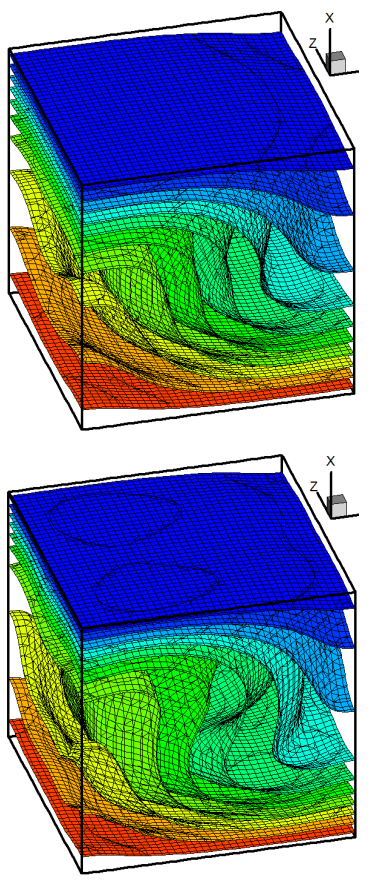

With Barrier, $\mathrm{H}_{1}=0.25$
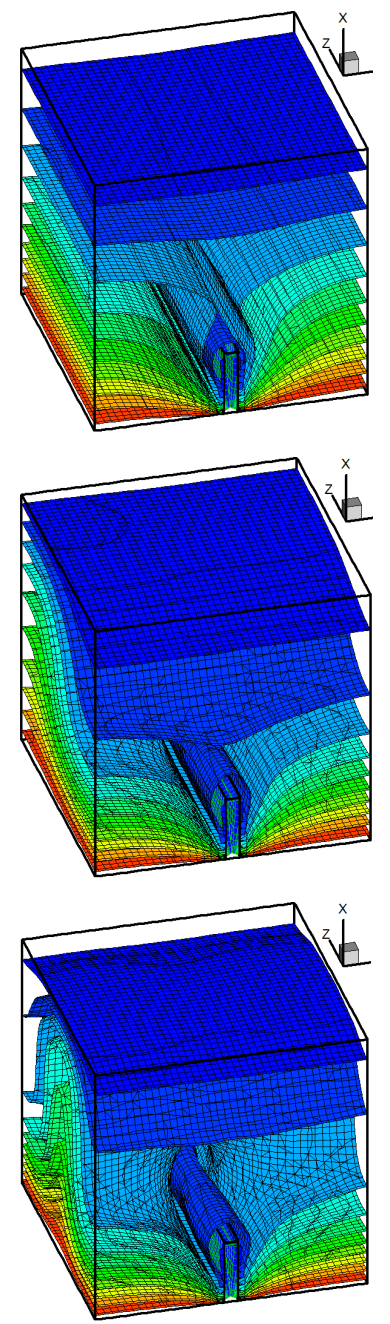

With Barrier, $\mathrm{H}_{2}=0.5$
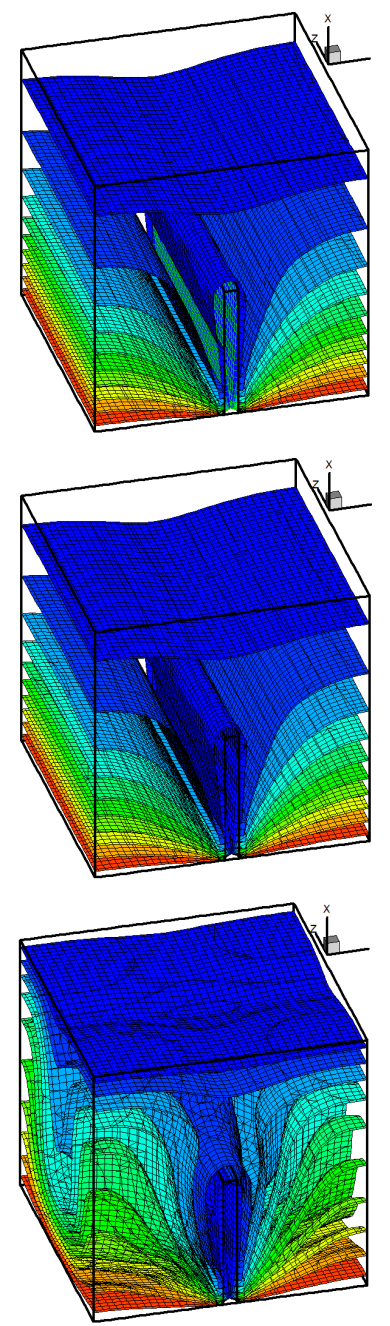

Figure 10. Temperature iso-surfaces in the building for different $R a$, effect of two barriers' heights for $N=-0.2$.

\subsubsection{Effect of Rayleigh and Barrier Height for $N=0.2$}

When the contaminant is a volatile organic compound (VOCs), then its emission and dispersion are caused by its volatilization having an ascendant effect. In this case, the thermal and solutal volume forces act in the same direction. This situation is modeled by a positive volume force ratio, which is the subject of this section. In this section, the flow structure, iso-concentration and iso-temperatures in the case of $N=0.2$ are presented.

Figure 11 shows the effect of the barrier on the particle trajectory for three Rayleigh values. When the cavity is barrier-free, the flow structure is characterized by a vortex encompassing the cavity in the $\mathrm{X}-\mathrm{Y}$ plane and rotating counterclockwise. Increasing the Rayleigh number increases the magnitude of the flow velocity. In general, and for all Rayleigh number values, the installation of the barrier decreases the flow velocity in the cavity. It can also be noticed that barrier 2 has a more pronounced effect on the flow attenuation. For $R a=10^{3}$, for both cases of barrier height, the flow structure is characterized by two vortexes in the $\mathrm{X}-\mathrm{Y}$ plane separated by the barrier and turning in the opposite direction. 
Ra

$10^{3}$

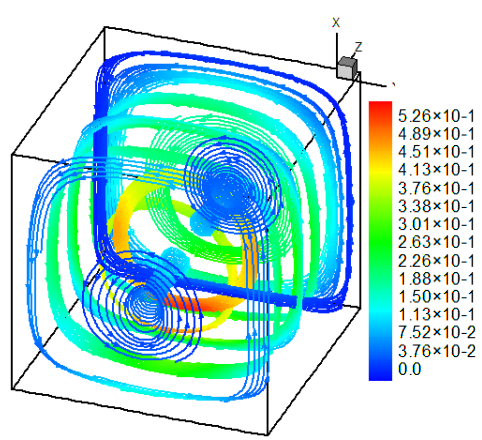

$10^{4}$

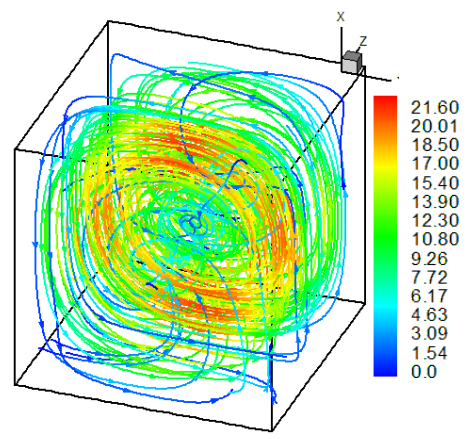

$2 \times 10^{4}$

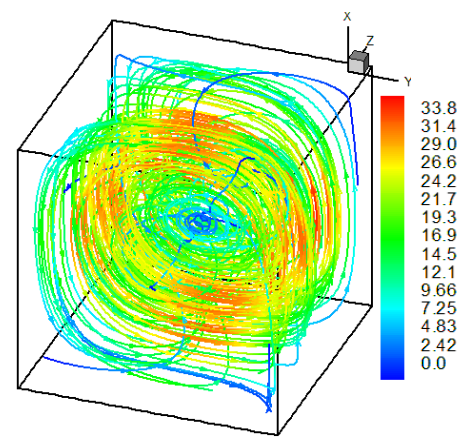

With Barrier, $\mathrm{H}_{1}=\mathbf{0 . 2 5}$
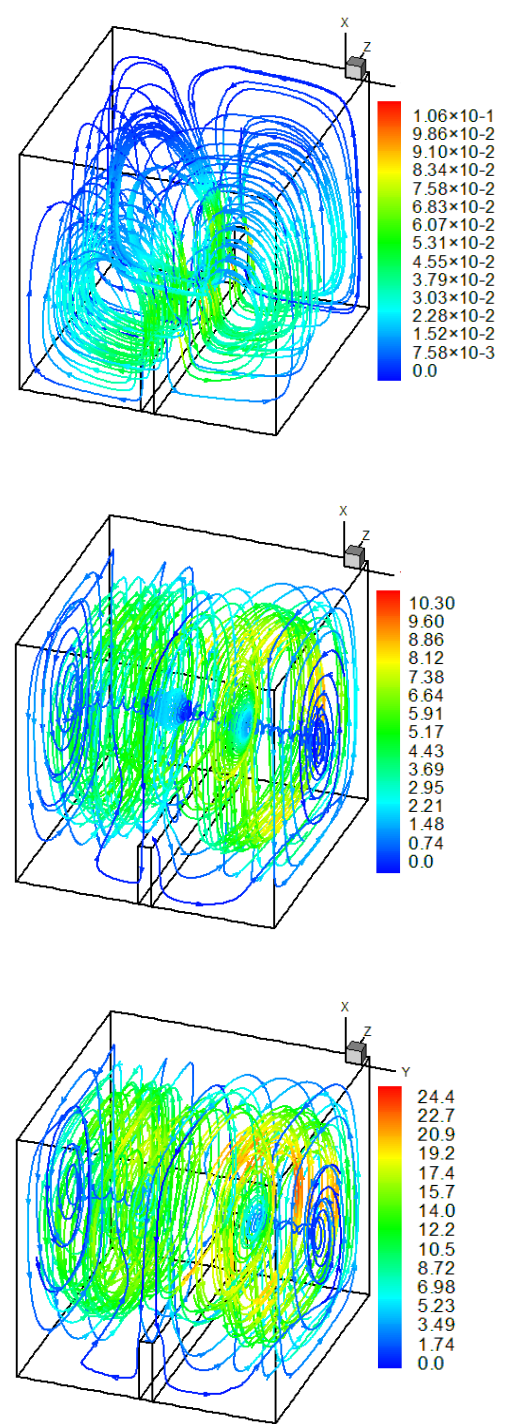
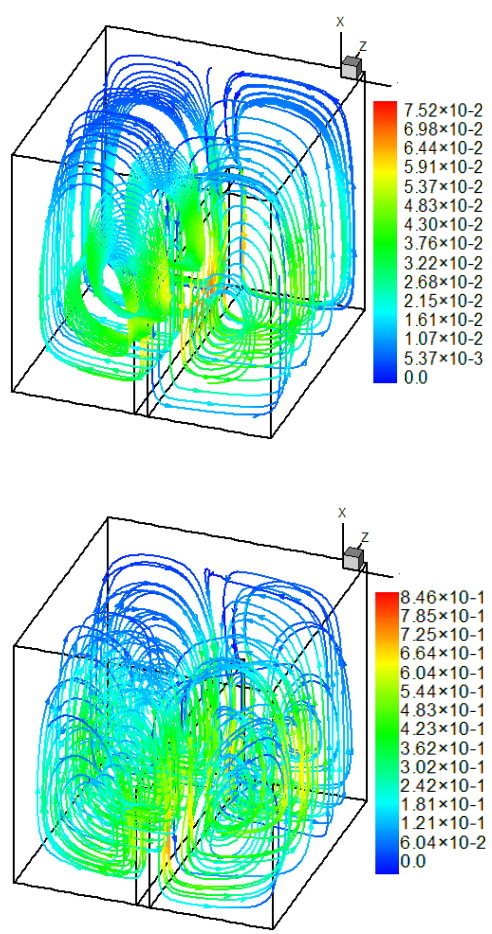

With Barrier, $\mathrm{H}_{2}=0.5$

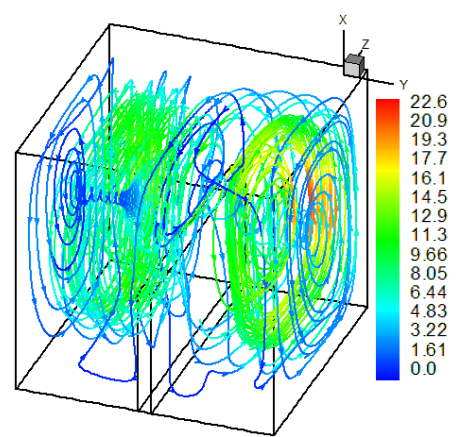

Figure 11. Particles' trajectories of the fluid in the building for different $R a$, effect of two barriers' heights for $N=0.2$.

A modification of the flow structure is observed for barrier 1 (the barrier with a height equal to $\mathrm{H}_{1}$ ) from $\mathrm{Ra}=10^{4}$ and for barrier 2 when $\mathrm{R} a=2 \times 10^{4}$. In fact, the flow structure is characterized by a vortex with spiral character carried in the $\mathrm{X}-\mathrm{Z}$ plane and turning counterclockwise.

It can also be noticed that in the presence of the barrier, the increase of the Rayleigh number has a double effect on the change of the flow structure and on the velocity magnitude. However, the increase of the velocity intensity is correlated to the direction and the plane of the vortex rotation. For barrier 1, it can be noticed that when $R a$ increases from $10^{3}$ to $10^{4}$, there is a change in the flow structure where the vortexes become located in the $X-Y$ plane and a high increase in the velocity magnitude of up to 100 times occurs. The velocity magnitude is only 10 times in the case of barrier 2 where the flow structure has not undergone a great change. As $R a$ increases from $10^{4}$ to $2 \times 10^{4}$, the change in the plane of rotation of the vortexes can be observed for barrier 2 and is associated with an increase in the velocity magnitude by 30 times. However, this increase is only by 2 times in the case of barrier 1 where the flow structure has not changed.

This correlation between the change of the plane carrying the vortices and the velocity magnitude can also be observed in the case of $N=-0.2$ (Figure 8) where the impact of the velocity increase is of the order of 15 times. This correlation can be observed for $R a=1.5 \times 10^{4}$ 
in barrier 1 and $R a=2 \times 10^{4}$ in barrier 2 . These observations show that the flow has a strong three-dimensional character.

Figure 12 shows the effect of the barrier and Rayleigh number on the contaminant iso-surfaces of concentration. For $R a=10^{3}$, the iso-concentrations are parallel, showing a dominant diffusive regime. The barrier has no significant effect on the solutal gradient. The height 2 allows an isolation of the dispersion of the concentration towards the left zone. The last finding is also observed when $R a=10^{4}$. However, from this number of $R a=10^{4}$, a convective solutal regime is noticed for the reference case, and the variation of the height of the barrier only affects the solutal gradient for $R a=10^{4}$.

Ra

$10^{3}$

$10^{4}$

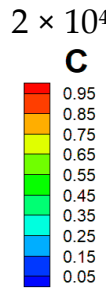

Reference case, No Barrier
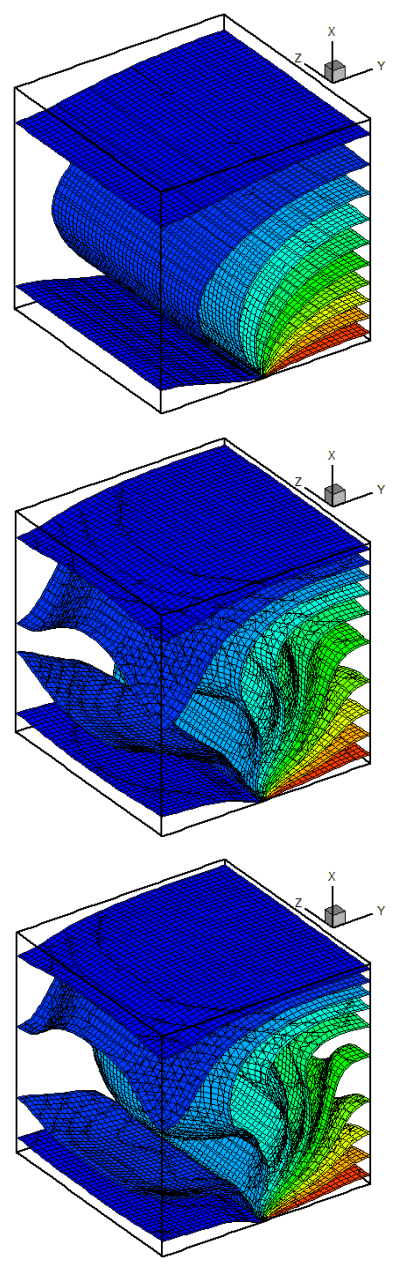

With Barrier, $\mathrm{H}_{1}=0.25$
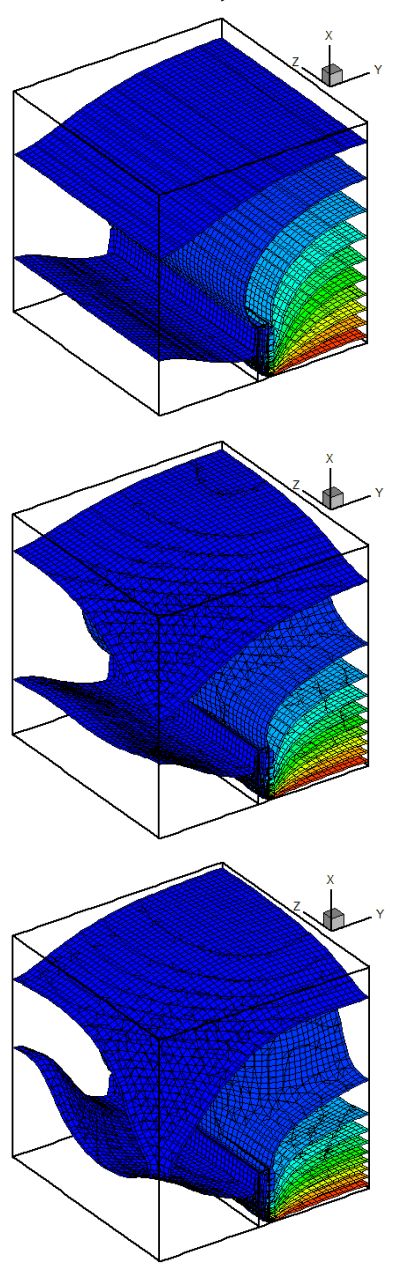

With Barrier, $\mathrm{H}_{2}=0.5$
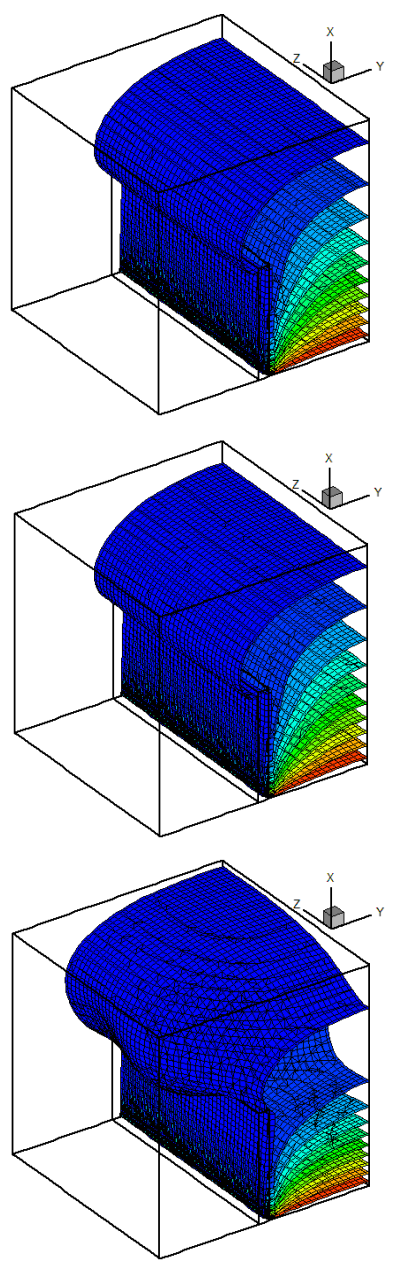

Figure 12. Contaminant iso-surfaces of concentration for different $R a$, effect of two barriers' heights for $N=0.2$.

Figure 13 shows the isotherms for the case $N=0.2$. It can be seen that the thermal convective effect appears in the cavity from $R a=10^{4}$. The presence of the barrier has caused a change in the isotherm structure. Indeed, the variation of the thermal gradient is initially a function of $y$, though it becomes a variable according to $z$. It can also be observed that the thermal gradient is weaker on the side of the contaminant source compared to the other side. 
Ra

$10^{3}$

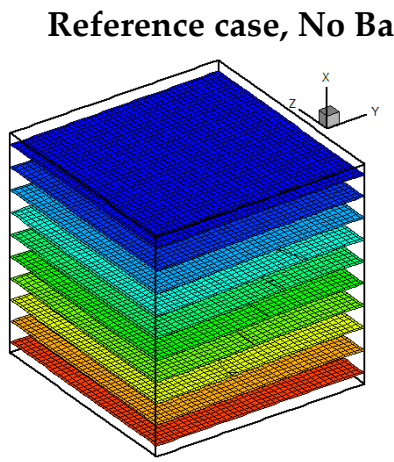

$10^{4}$

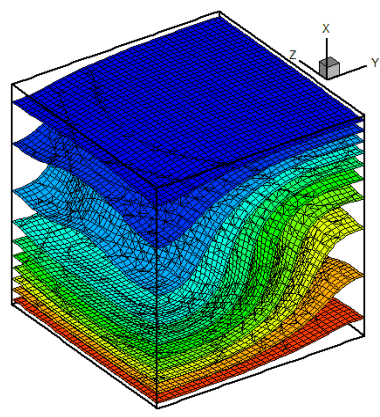

$2 \times 10^{4}$
$\mathbf{T}$
$\mathbf{T}$
0.95
0.85
0.75
0.65
0.55
0.45
0.35
0.25
0.15
0.05

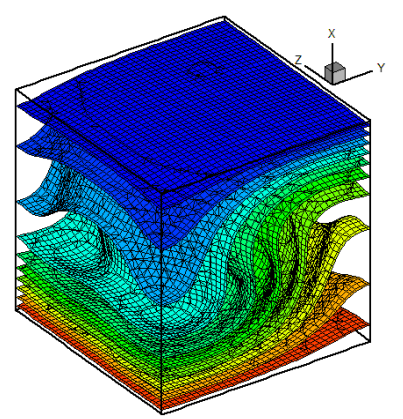

With Barrier, $\mathrm{H}_{1}=0.25$
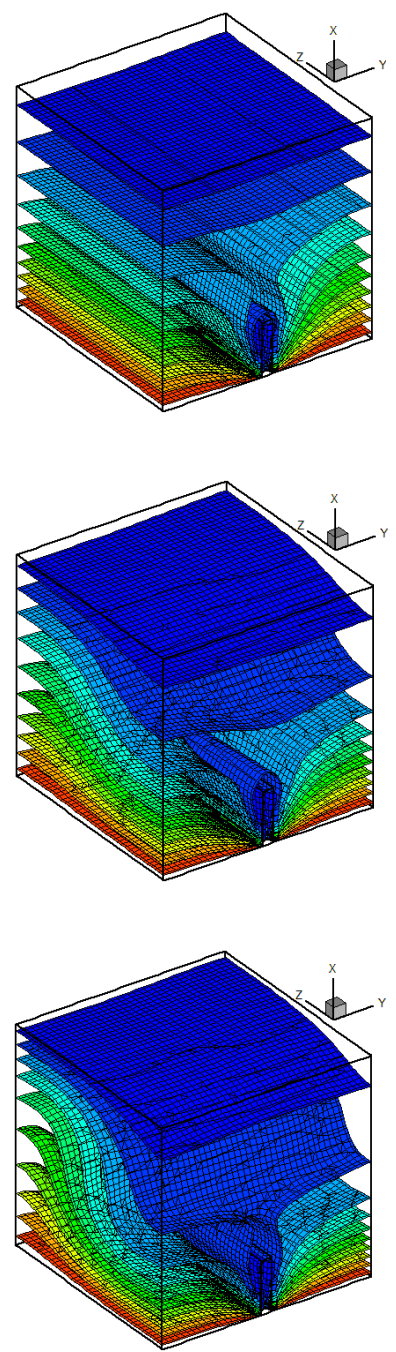
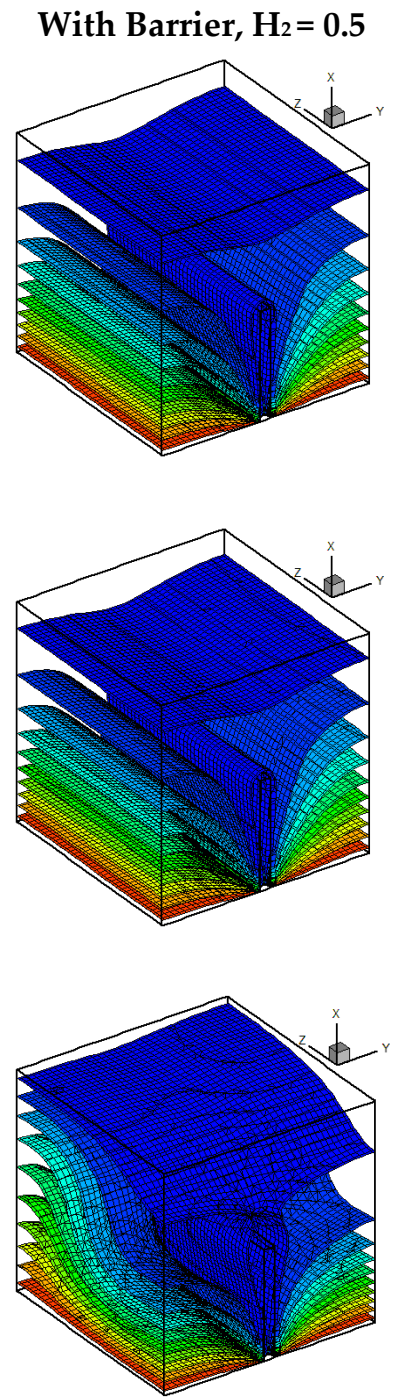

Figure 13. Temperature iso-surfaces in the building for different $R a$, effect of two barriers' heights for $N=0.2$.

\subsection{Efficiency of Protective Barrier and Heat-Mass Transfer Ratios}

\subsubsection{Barrier Efficiency}

To study the efficiency of the barrier in reducing the dispersion of the contaminant from the region containing the contaminant source to the region on the left, the analysis focused on the evaluation of the concentration of the contaminant in the plane $y=0.45$, where the plane is very close to the limit of the contamination surface (Figure 14). In this plane, we looked for the maximum contaminant concentration observed in the case of a building with and without a barrier. It can be noticed that the coordinates of the maximum concentration depend on the buoyancy ratio, Rayleigh number and time. For the positive buoyancy ratio, the maximum concentration is located at the top, but for the negative value, it is located at the bottom, and the concentration distributions have a three-dimensional character. 

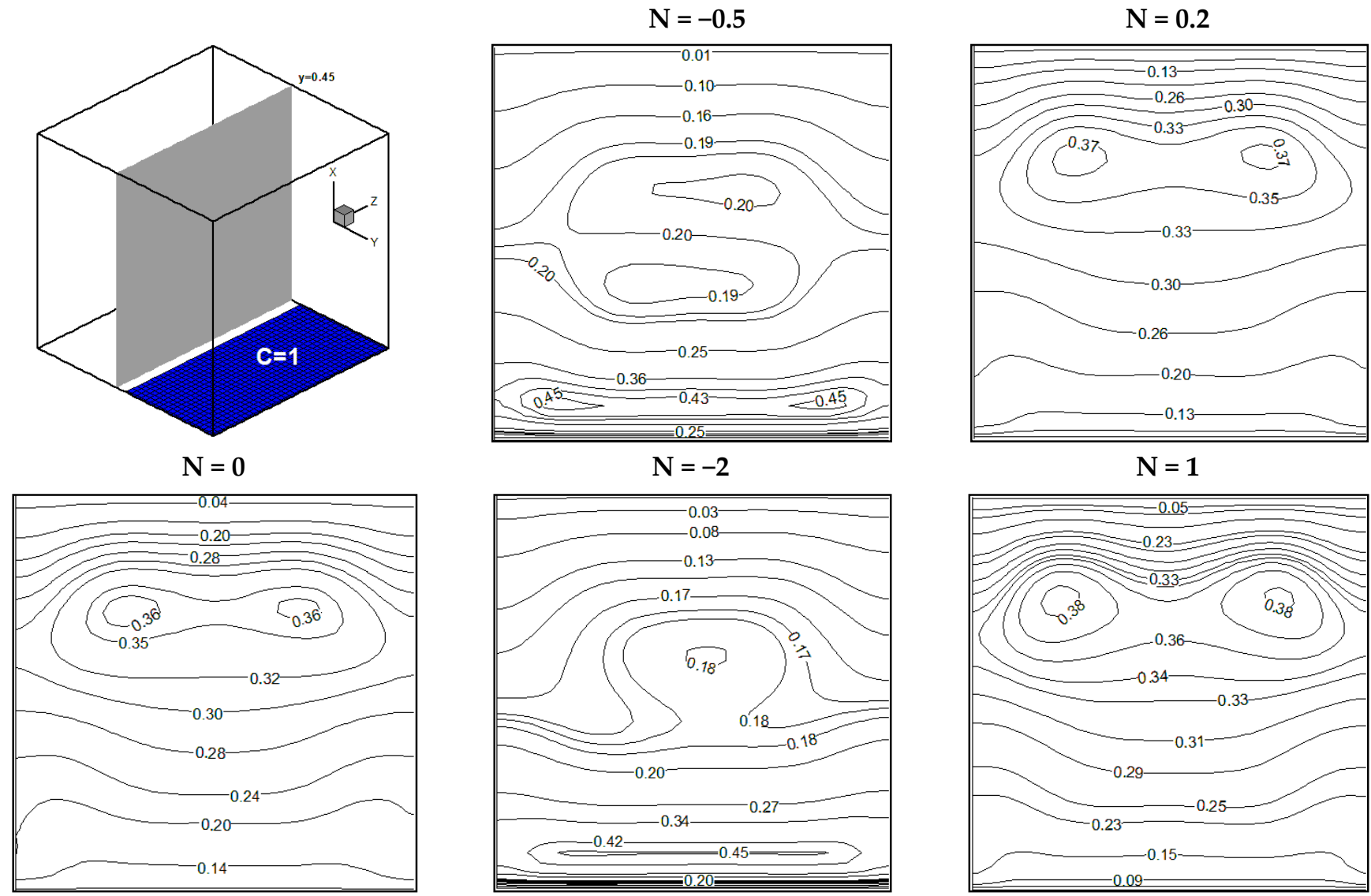

Figure 14. Iso-contours of the contaminant concentration in the building without a barrier, with the $y=0.45$ plane for $R a=2 \times 10^{4}$ and a different buoyancy ratio.

The barrier efficiency is defined by this relation $E_{f f}=1-\frac{c_{\max }}{C_{\max }}$, where $c_{\max }$ is the maximum contaminant concentration in the plane $y=0.45$, for the building with a barrier. Also, $c_{\max 0}$ is the maximum contaminant concentration in the plane $y=0.45$, for the reference building without a barrier.

Figure 15 shows the effect of the Rayleigh number on the efficiency of the barrier for two heights $\mathrm{H}_{1}=0.25$ and $\mathrm{H}_{2}=0.5$. A minimum efficiency equal to $36.22 \%$ can be observed for height $1, R a=10^{4}$ and $N=0.2$. Meanwhile, the maximum efficiency $(85.18 \%)$ can be observed for height $2, R a=1.5 \times 10^{4}$ and $N=-0.2$. For height 1 and when $N=0.2$, the increase in $R a$ decreases the efficiency up to $R a=10^{4}$, reaching a minimum, then the increase in efficiency takes on a positive slope, and the percentages increase with the increase of Ra. When $N=-0.2$, it can be observed that going from $R a=10^{3}$ to $10^{4}$, the efficiency increases from $50 \%$ to $60 \%$ and then decreases to $52 \%$ for $R a=1.5 \times 10^{4}$ before it resumes its positive slope and reaches more than $75 \%$ for $R a=10^{5}$.

For height 2 and when $N=0.2$, the increase of $R a$ from $10^{3}$ to $10^{4}$ has no effect on the efficiency of the barrier and it is starting from $R a=1.5 \times 10^{4}$ that we can observe a decrease reaching a minimum equal to $64 \%$, which was observed for $R a=2 \times 10^{4}$. From this value, an increase in $R a$ increases the efficiency. When $N=-0.2$, a slight increase in efficiency is observed from $R a=10^{3}$ to $1.5 \times 10^{4}$, reaching a maximum equal to $85.18 \%$. From this value, the increase in $R a$ values decreases the efficiency. 


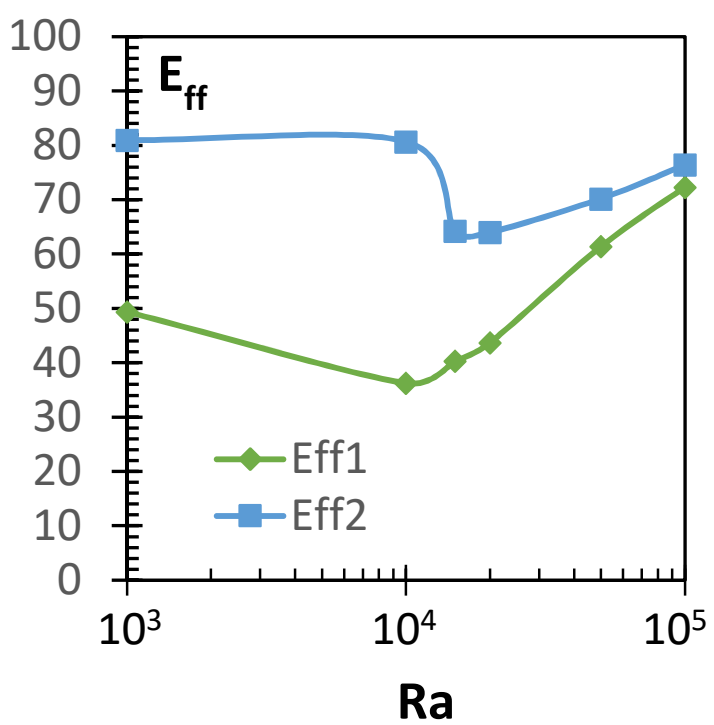

$\mathrm{N}=0.2$

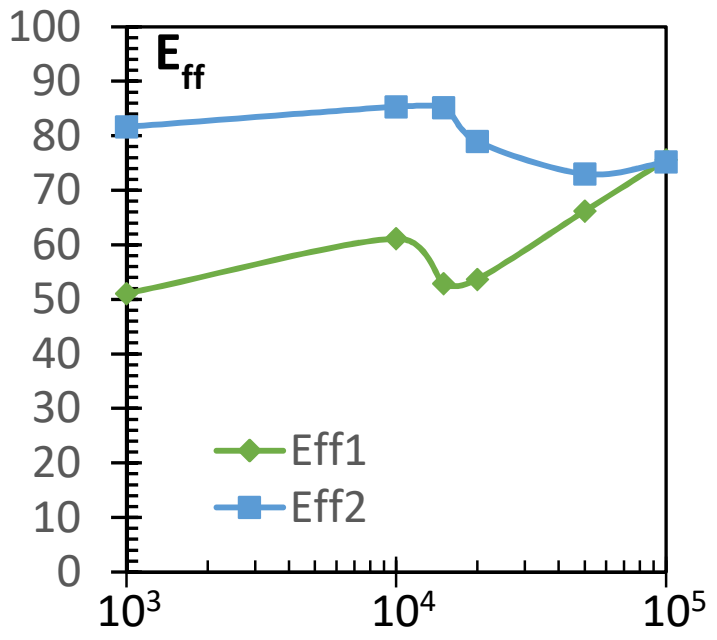

Ra

$$
\mathrm{N}=-0.2
$$

Figure 15. Effect of the Rayleigh number on the efficiency of the barrier for $N=0.2$ and $N=-0.2$; Eff 1 and Eff 2 are the barrier efficiencies for $\mathrm{H}_{1}=0.25$ and $\mathrm{H}_{2}=0.5$, respectively.

Figure 16 shows the effect of the buoyancy ratio on the efficiency of the barrier when $R a=10^{4}$. It can be observed that the increase in the efficiency as a function of $N$ is similar for the two heights of the barrier $\mathrm{H}_{1}$ and $\mathrm{H}_{2}$. The efficiency is $85 \%$ for $\mathrm{H}_{2}$ and all negative values of $N$ under -0.5 , and it is $57 \%$ for $\mathrm{H}_{1}$. From this value of $N=-0.5$, a decrease in the efficiency can be noticed, reaching a minimum equal to $41.67 \%$ at $\mathrm{N}=0$ for $\mathrm{H}_{1}$ and $62.96 \%$ at $N=0.5$ for $\mathrm{H}_{2}$. Then the increase in the efficiency takes on a positive slope with the increase of $\mathrm{N}$. The barrier efficiency percentages are higher for negative buoyancy ratios than for positive ones.

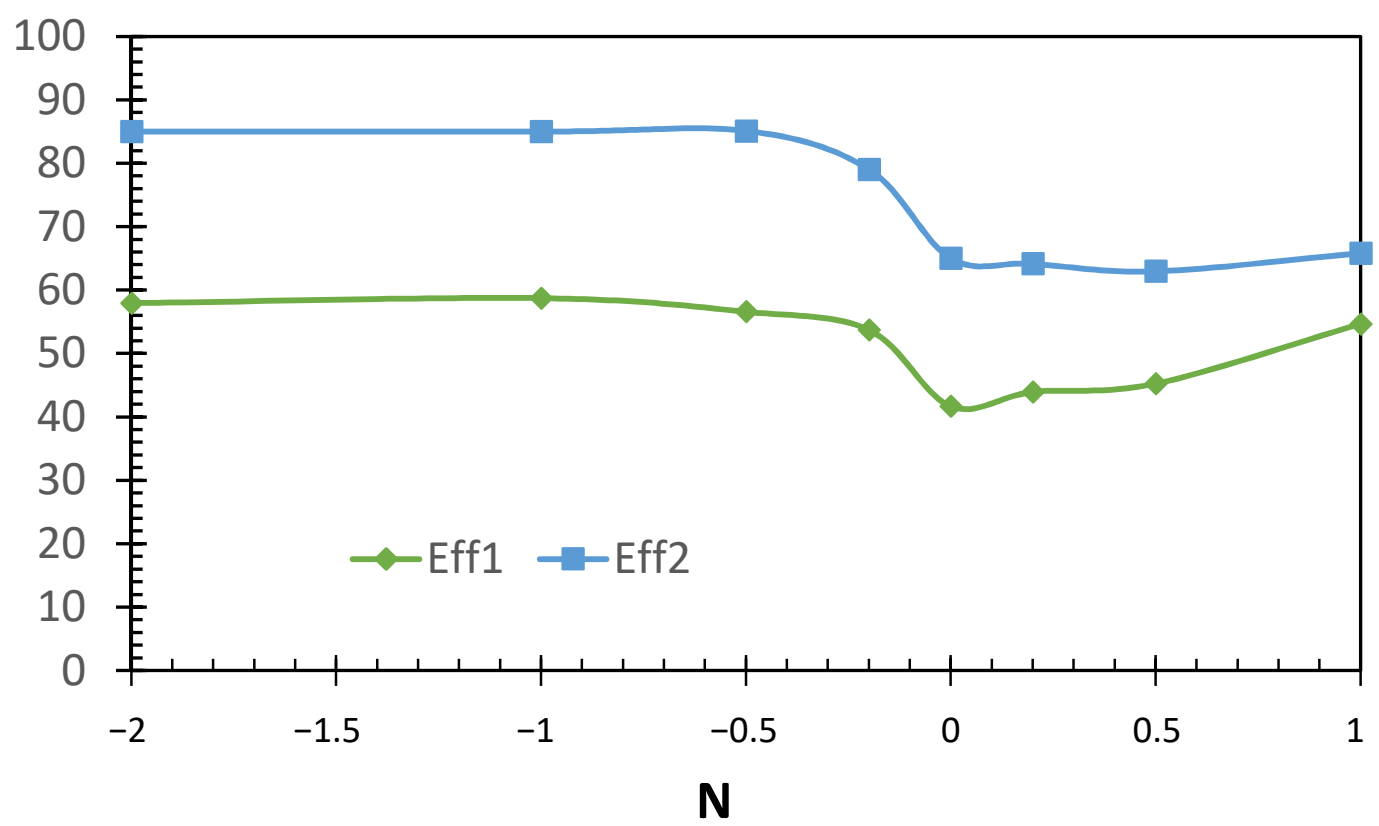

Figure 16. Effect of the buoyancy ratio on the efficiency of the barrier for $R a=2 \times 10^{4}$; Eff 1 and Eff 2 are the efficiencies for $\mathrm{H}_{1}=0.25$ and $\mathrm{H}_{2}=0.5$, respectively. 
Figure 17a shows the effect of the barrier height on efficiency for $R a=2 \times 10^{4}$, $N=0.2$ and $N=-0.2$. For all values of $\mathrm{H}$, the efficiencies for $\mathrm{N}=-0.2$ are higher than those for $N=0.2$. For $N=0.2$, the trajectory can be divided into two parts; from $\mathrm{H}=0.12$ to $\mathrm{H}=0.35$, the slope of the curve is 111.61 , but from $\mathrm{H}=0.35$ to 0.75 , the slope decreases and becomes 81.96 .

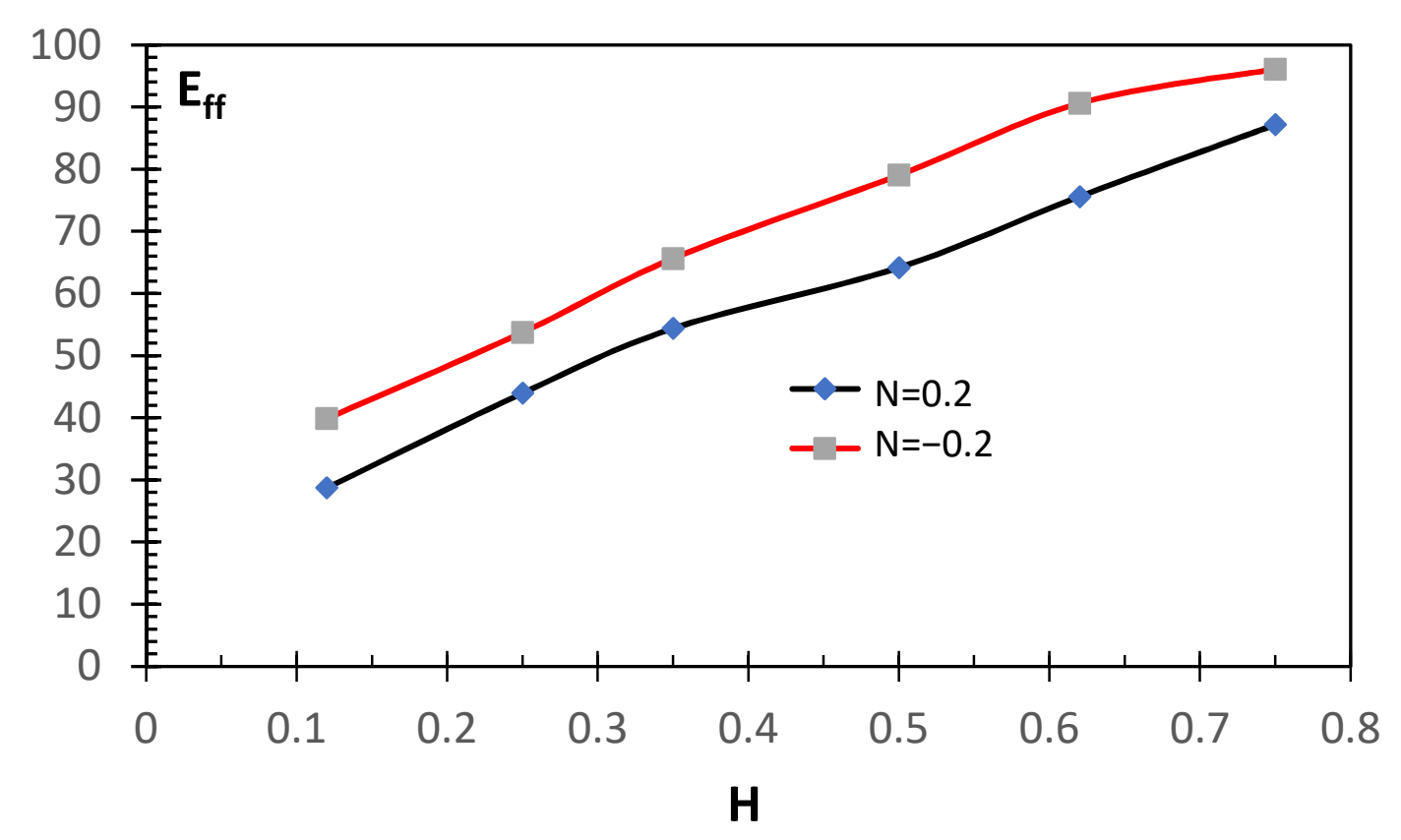

(a)

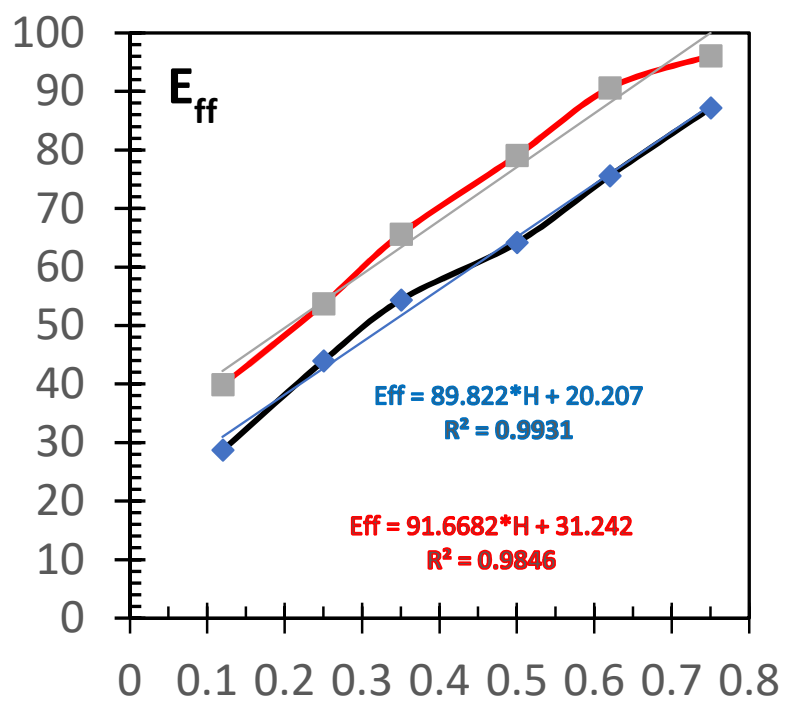

H

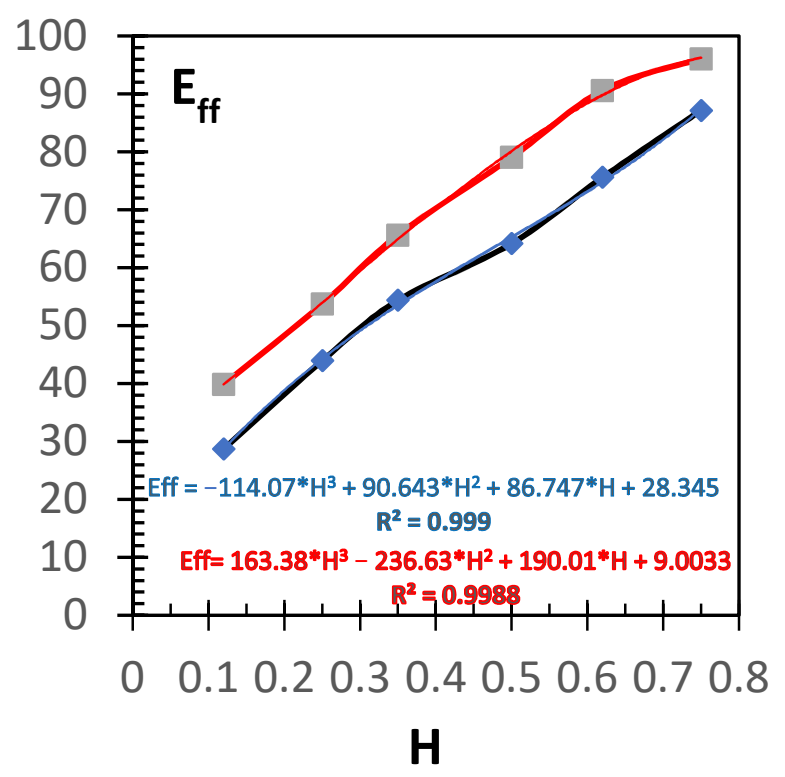

(c)

Figure 17. (a) Effect of the barrier height on the efficiency for $R a=2 \times 10^{4}, N=0.2$ and $N=-0.2$; (b) Linear trendline to efficiency variation with the barrier height; (c) Polynomial trendline to efficiency variation with the barrier height, blue color equation for $N=0.2$ and red color equation for $N=-0.2$. 
For $N=-0.2$, the efficiency pattern follows three slopes: the highest one is from $\mathrm{H}=0.12$ to 0.35 , and it is 112.02 , the second one is from 0.35 to 0.62 and it is 76.15 and the third one is the lowest one of 42.19 from 0.62 to 0.75 . In this latest range, the efficiency goes from $90 \%$ to $96 \%$.

To further analyze the efficiency variation as a function of height, the two curves for $N=0.2$ and $N=-0.2$ are treated by adding a trendline (Figure $17 \mathrm{~b}, \mathrm{c}$ ). Two forms of equations are examined: linear (Figure $17 \mathrm{~b}$ ) and polynomial (Figure 17c) and the $R^{2}$ trendline reliability for each equation is compared. It can be found that the polynomial variation of order 3 gives a better reliability trendline, closer to 1 . So, we can conclude that the efficiency increases following a polynomial evolution of order 3 as $E_{f f}=C_{1} H^{3}+$ $\mathrm{C}_{2} \mathrm{H}^{2}+\mathrm{C}_{3} \mathrm{H}+\mathrm{C}_{4}$ and that the constants $\mathrm{C}_{1}, \mathrm{C}_{2}, \mathrm{C}_{3}$ and $\mathrm{C}_{4}$ depend on the Rayleigh and $\mathrm{N}$ numbers.

\subsubsection{Heat and Mass Transfer Ratios}

Figure 18 shows the effect of the buoyancy ratio on the heat and mass transfer ratio for $R a=2 \times 10^{4}$ for three cases: (0) considered as a reference without a barrier, (1) case of barrier height $\mathrm{H}_{1}$ and (2) for $\mathrm{H}_{2}$. It can be noticed that the presence of the barrier decreases the heat and mass transfer rates. The average Nusselt number in the reference case increases with two slopes. An increase in the slope of the average Nusselt number is observed from $N=0$. For a barrier of height $H 1$, the variation is parabolic with a maximum at $N=0.5$. The average Nusselt number for the case of height 2 with a minimum variation is observed at $N=-0.5$ while the maximum occurs at $N=0.5$. For a negative buoyancy ratio values, the increase in $N$ has no significant effect on the average Sherwood number for the different cases studied. From $N=-0.2$ onwards, an increase in the mass transfer rate is noticed.
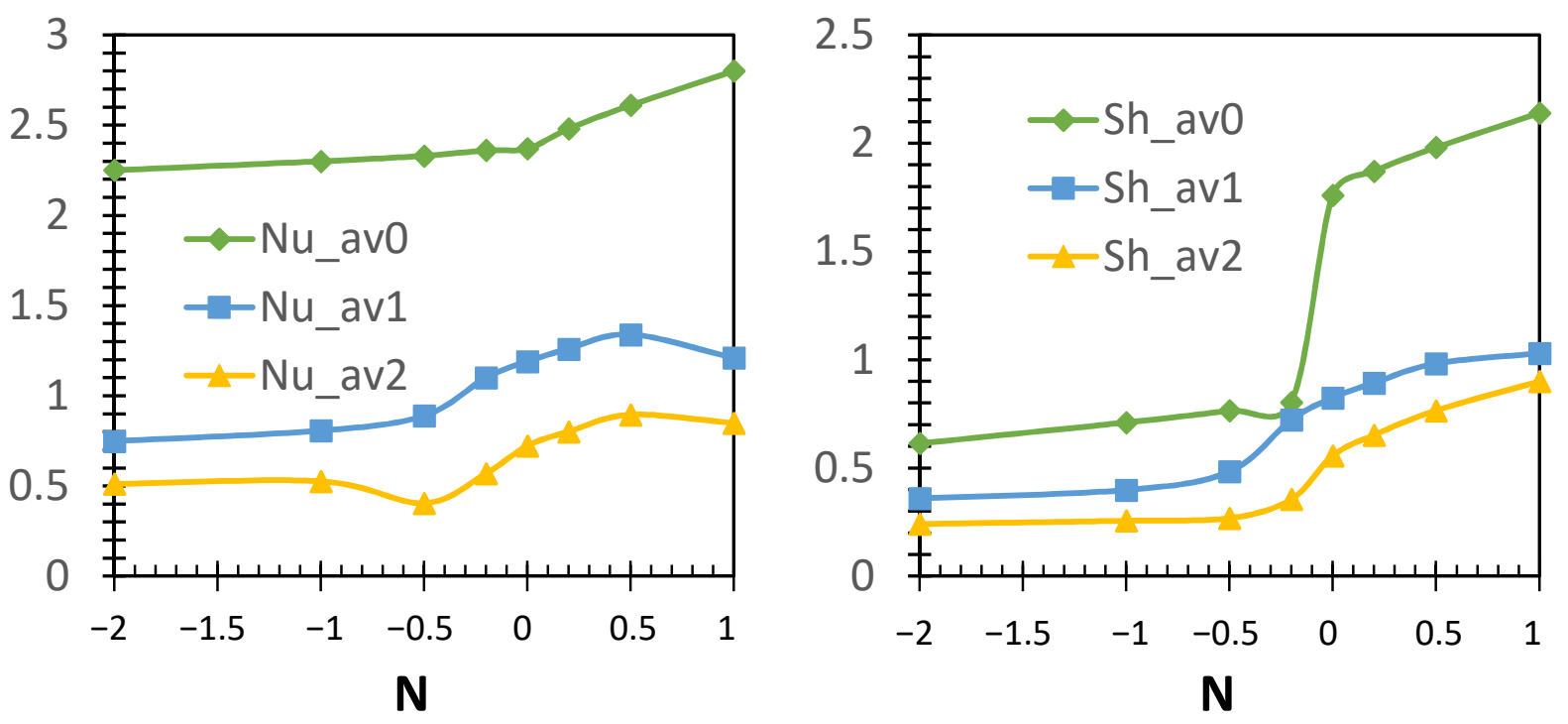

Figure 18. Effect of the buoyancy ratio on the average heat and mass transfer ratios for $R a=2 \times 10^{4},(0)$ : reference case, (1): $\mathrm{H}_{1}=0.25$ and (2) for $\mathrm{H}_{2}=0.5$.

Figure 19 presents the effect of the Rayleigh number on the average Sherwood number for $N=0.2$ and $N=-0.2$. When $N=0.2$, there is a linear increase in the average Sherwood number as a function of $R a$ for the reference case. When the barrier is placed, the average Sherwood number changes in two incremental steps. When $R a$ is less than $10^{4}$, a slight increase in the mass transfer rate is observed. From this value, the increase of average Sherwood number is more than double when $R a=10^{5}$. 


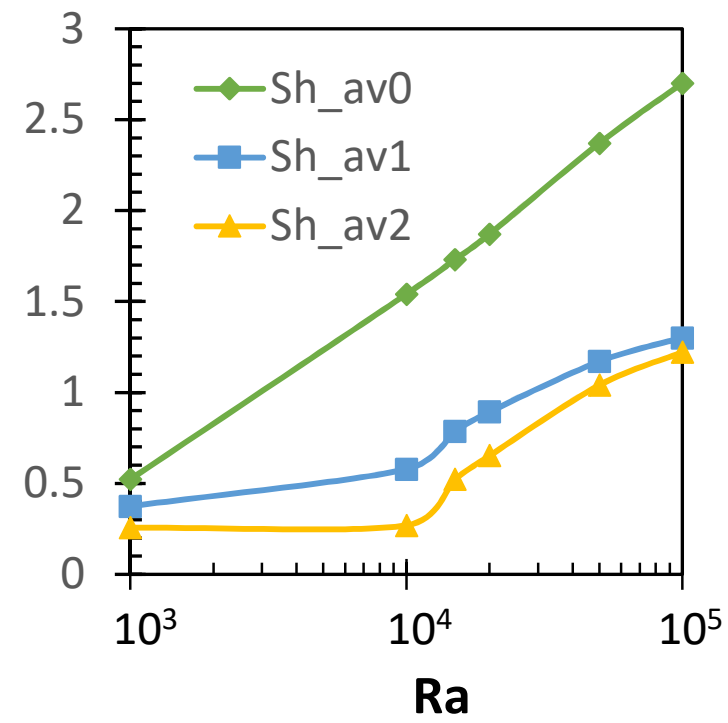

$\mathrm{N}=0.2$

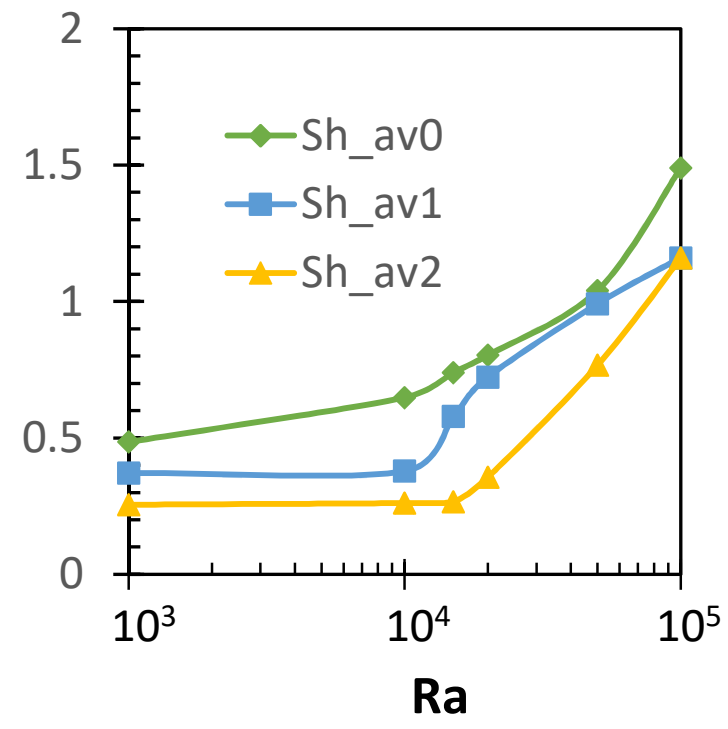

$\mathrm{N}=-0.2$

Figure 19. Effect of the Rayleigh number on the average mass transfer ratios for $N=0.2$ and $N=-0.2,(0)$ : reference case, (1): $\mathrm{H}_{1}=0.25$ and (2) for $\mathrm{H}_{2}=0.5$.

When $N=-0.2$, the trajectory of the average Sherwood number variation is similar for the different cases studied. From $R a=10^{3}$ to $10^{4}$, the mass transfer rate is almost constant with a slight increase observed for the reference case. From $R a=10^{4}$, the increase in $R a$ significantly increases the average Sherwood number for the three cases studied. For $R a=5 \times 10^{4}$, it can be observed that the average Sherwood number for the reference case is nearly equal to that for the $\mathrm{H}_{1}$.

Figure 20 shows the effect of the Rayleigh number on the average heat transfer rate. The increase in $R a$ increases the average Nusselt number almost linearly in the reference case for both buoyancy ratio cases. For the cavity with barriers, the $R a$ increases from $10^{3}$ to $10^{4}$, and the average Nusselt is almost constant. From $R a=10^{4}$, the increase in $R a$, significantly increases the heat transfer ratio for both heights of the barrier studied when $N=0.2$. For $N=-0.2$, the case of the barrier of height 1 is characterized by a maximum average Nusselt number observed at $R a=5 \times 10^{4}$. For the case of the barrier of height 1 , the increase of the Nusselt number is observed from $R a=1.5 \times 10^{4}$.

Figure 21 presents the effect of barrier height on the average mass and heat transfer ratios for $R a=2 \times 10^{4}, N=0.2$ and $N=-0.2$. Two phases can be observed for the decrease of the average Sherwood number when $N=0.2$. A decrease of $180 \%$ is detected from $\mathrm{H}=0$ to 0.12 , then the curve takes a lower negative slope and the decrease is of the order of $50 \%$ from 0.12 to 0.75 . When $N=-0.2$, there is a slight increase in the average Sherwood number from $\mathrm{H}=0$ to $\mathrm{H}=0.12$ and as the height of the barrier increases, the average mass transfer rate decreases as well.

It can also be noticed that the increase in the height of the barrier decreases the average Nusselt number. Starting from 0 to 0.35 , the average Nusselt number decreases by $250 \%$. Then by passing from $\mathrm{H}=0.35$ to 0.75 , the decrease becomes about $50 \%$. 


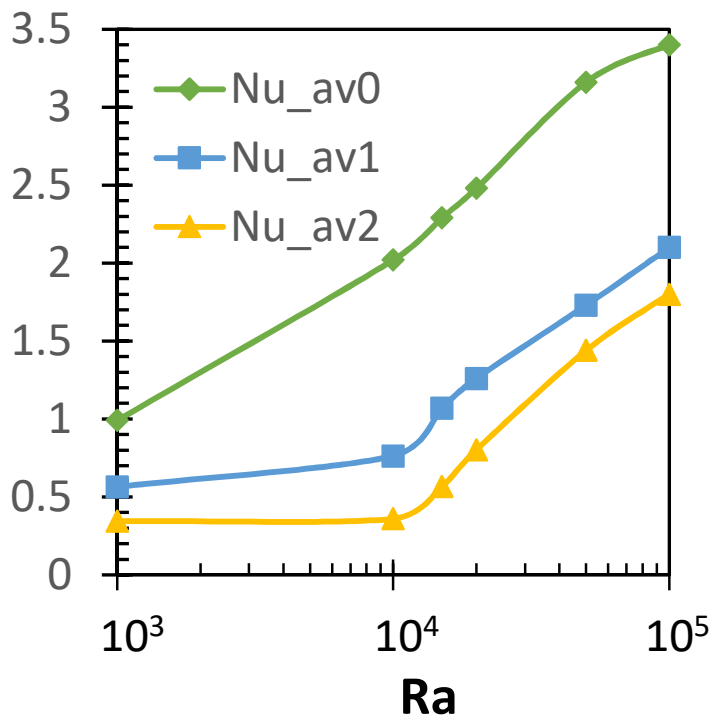

$\mathrm{N}=0.2$

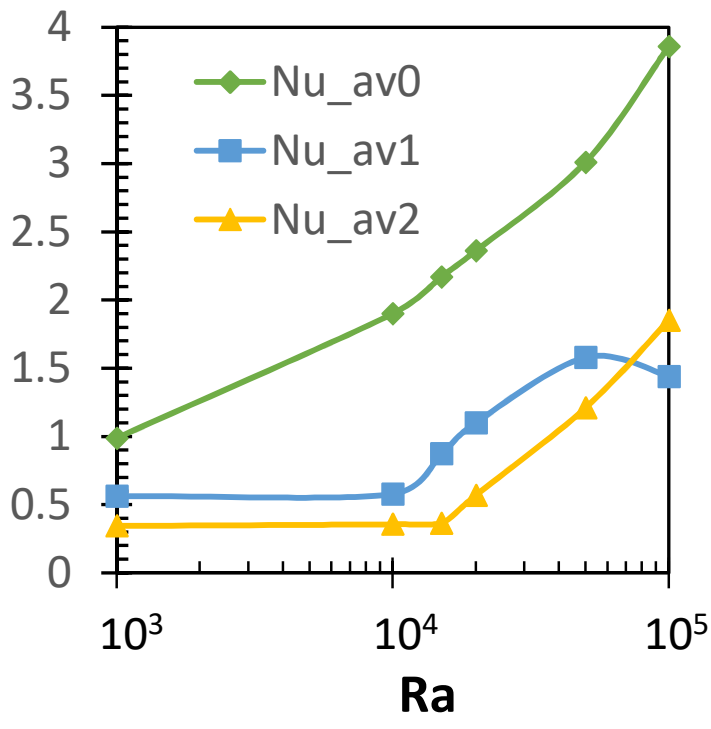

$\mathrm{N}=-0.2$

Figure 20. Effect of the Rayleigh number on the average heat transfer ratios for $N=0.2$ and $N=-0.2,(0)$ : reference case, (1): $\mathrm{H}_{1}=0.25$ and (2) for $\mathrm{H}_{2}=0.5$.
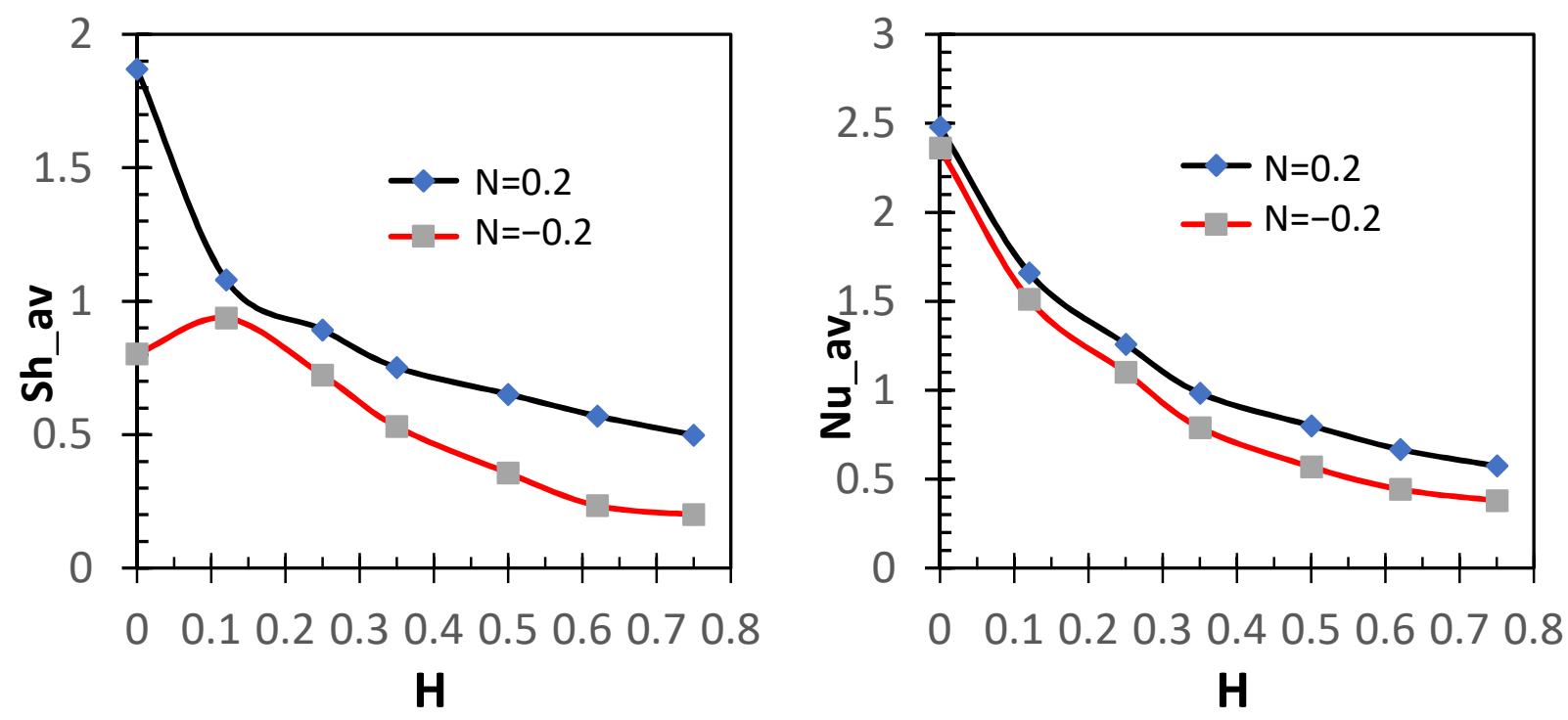

Figure 21. Effect of the barrier height on the average heat and mass transfer ratios for $R a=2 \times 10^{4}, N=0.2$ and $N=-0.2$.

\section{Conclusions}

The study presented in this paper focuses on the impact of placing an adiabatic impermeable barrier on the local dispersion of an aero-contaminant inside a three-dimensional cavity subjected to the Rayleigh-Benard conditions.

In the first part of this paper, the effect of different parameters on the dispersion of the contaminant in the case of a cavity without a barrier is analyzed. In the second part, the effect of the Rayleigh number and barrier height on the flow structure is studied through iso-concentration and iso-temperature plots for different values of $\mathrm{N}: 0,-0.2$ and 0.2 .

In the third part, a parametric study on the effects of the Rayleigh number, the buoyancy ratio and the barrier height on the effectiveness of the barrier in the reduction of the cavity contamination, as well as the heat and mass transfer rates, is performed.

The most important findings can be summarized as follows: 
- In the absence of a barrier, the dispersion of the contaminant is mainly directed upwards at the corners of the building and to the left in the core of the building to the initially uncontaminated region, in the cases of $N=0$ and $N$ positive. Otherwise, when $N$ is negative, the dispersal effect of the contaminant mainly occurs in a horizontal way at the bottom of the building.

- The effectiveness of the protective barrier is greater in the case of negative buoyancy ratios compared to positive values, for the same Rayleigh number and the same barrier height.

- The efficiency increases linearly with the height of the barrier.

- In the presence of the barrier, the Rayleigh number increase causes the heat and mass transfer rates to increase from $R a=10^{4}$ and higher.

- The placement of the barrier strongly diminishes the heat transfer rate for all values of N. However, noticeable decrease in the mass transfer rate is only observed for positive values of $\mathrm{N}$.

Funding: This research received no external funding.

Institutional Review Board Statement: Not applicable.

Informed Consent Statement: Not applicable.

Data Availability Statement: Not applicable.

Conflicts of Interest: The author declares no conflict of interest.

\section{References}

1. Godish, T. Indoor Environmental Quality, 1st ed.; CRC Press: Boca Raton, FL, USA, 2000; pp. 230-250.

2. Cogliano, V.J.; Grosse, Y.; Baan, R.A.; Straif, K.; Secretan, M.B.; El Ghissassi, F. Meeting Report: Summary of IARC Monographs on Formaldehyde, 2-Butoxyethanol, and 1-tert-Butoxy-2-Propanol. Enviro. Health Prespec. 2005, 113, 1205-1208. [CrossRef]

3. Branco, P.T.B.S.; Alvim-Ferraz, M.C.M.; Martins, F.G.; Sousa, S.I.V. Children's exposure to indoor air in urban Nurseries-Part II: Gaseous pollutants' assessment. Environ. Res. 2015, 142, 662-670. [CrossRef] [PubMed]

4. Little, J.C.; Hodgson, A.T.; Gadgil, A.J. Modeling emissions of volatile organic compounds from new carpets. Atmosph. Environ. 1994, 28, 227-234. [CrossRef]

5. Yang, X.; Chen, Q.; Zhang, J.S.; Magee, R.; Zeng, J.; Shaw, C.Y. Numerical simulation of VOC emissions from dry materials. Build. Environ. 2001, 36, 1099-1107. [CrossRef]

6. Guo, Z. Review of indoor emission source models. Part 1. Overview. Environ. Pollut. 2002, 120, 533-549. [CrossRef]

7. Xu, Y.; Zhang, Y. An improved mass transfer-based model for analyzing VOC emissions from building materials. Atmosph. Environ. 2003, 37, 2497-2505. [CrossRef]

8. Deng, B.; Kim, N. An analytical model for VOCs emission from dry building materials. Atmosph. Environ. 2004, 38, 1173-1180. [CrossRef]

9. Wiglusz, R.; Sitko, E.; Nikel, G.; Jarnuszkiewicz, I.; Igielska, B. The effect of temperature on the emission of formaldehyde and volatile organic compounds (VOCs) from laminate flooring-Case study. Build. Environ. 2002, 37, 41-44. [CrossRef]

10. Xiong, J.; Zhang, P.; Huang, S.; Zhang, Y. Comprehensive influence of environmental factors on the emission rate of formaldehyde and VOCs in building materials: Correlation development and exposure assessment. Environ. Res. 2016, 151, 734-741. [CrossRef] [PubMed]

11. Deng, Q.H.; Zhou, J.; Mei, C.; Shen, Y.M. Fluid, heat and contaminant transport structures of laminar double-diffusive mixed convection in a two-dimensional ventilated enclosure. Int. J. Heat Mass Transf. 2004, 47, 5257-5269. [CrossRef]

12. Yu-Shu, S.; Liu, D.; Wang, Y.; Zhao, F.; Li, Y. Forced flow structure and mixed convection in a ventilated porous enclosure with a local contaminant source. Int. J. Heat Mass Transf. 2019, 131, 973-983. [CrossRef]

13. Zhao, F.Y.; Liu, D.; Tang, G.F. Natural convection in an enclosure with localized heating and salting from below. Int. J. Heat Mass Transf. 2008, 51, 2889-2904. [CrossRef]

14. Arellano, J.S.; Gijón-Rivera, M.; Riesco-Ávila, J.M.; Elizalde-Blancas, F. Numerical study of the double diffusive convection phenomena in a closed cavity with internal $\mathrm{CO}_{2}$ point sources. Int. J. Heat Mass Transf. 2014, 71, 664-674. [CrossRef]

15. Li, X.; Yan, Y.; Tu, Y.J. Effects of surface radiation on gaseous contaminants emission and dispersion in indoor environment-A numerical study. Int. J. Heat Mass Transf. 2019, 131, 854-862. [CrossRef]

16. Abolhasani, M.; Abbasbandy, S.; Allahviranloo, T. A New Variational Iteration Method for a Class of Fractional ConvectionDiffusion Equations in Large Domains. Mathematics 2017, 5, 26. [CrossRef]

17. Geiser, J.; Martínez, E.; Hueso, J.L. Serial and Parallel Iterative Splitting Methods: Algorithms and Applications to Fractional Convection-Diffusion Equations. Mathematics 2020, 8, 1950. [CrossRef] 
18. Mallinson, G.D.; Davis, G.D.V. Three-dimensional natural convection in a box: A numerical study. J. Fluid Mech. 1977, 83, 1-31. [CrossRef]

19. Wakashima, S.; Saitoh, T.S. Benchmark solutions for natural convection in a cubic cavity using the high-order time-space method. Int. J. Heat Mass Transf. 2004, 47, 853-864. [CrossRef]

20. Eymard, R.; Gallouet, T.; Herbin, R. Finite Volume Methods, Handbook of Numerical Analysis; Elsevier BV: Amsterdam, The Netherlands, 2000; Volume 7, pp. 713-1020.

21. Patankar, S.V. Numerical Heat Transfer and Fluid Flow, 2nd ed.; Mc Graw Hill: New York, NY, USA, 1980; pp. 25-39.

22. Bejan, A. Convection Heat Transfer, 4th ed.; John Wiley: New York, NY, USA, 2013; pp. 30-289.

23. Sezai, I.; Mohamad, A.A. Double diffusive convection in a cubic enclosure with opposing temperature and concentration gradients. Phys. Fluids 2000, 12, 2210-2223. [CrossRef]

24. Rahmati, A.R.; Tahery, A.A. Numerical study of nanofluid natural convection in a square cavity with a hot obstacle using lattice Boltzmann method. Alexandria Eng. J. 2018, 57, 1271-1286. [CrossRef] 